\title{
1 A Near-Real-Time Approach for Monitoring Forest Disturbance 2 Using Landsat Time Series: Stochastic Continuous Change 3 Detection
}

\author{
Su Ye*1, John Rogan ${ }^{1}$, Zhe Zhu' ${ }^{2}$, J. Ronald Eastman ${ }^{3}$
}

\section{Abstract}

Forest disturbances greatly affect the ecological functioning of natural forests. Timely information regarding extent, timing and magnitude of forest disturbance events is crucial for effective disturbance management strategies. Yet, we still lack an acute, near-real-time and high-performance remote sensing tools for monitoring abrupt and subtle forest disturbances. This study presents a new approach called 'Stochastic Continuous Change Detection (S-CCD)' using a dense Landsat data time series. S-CCD improves upon the 'COntinuous monitoring of Land Disturbance (COLD)' approach by incorporating a mathematical tool called the 'state space model', which treats trends and seasonality as stochastic processes, allowing for modeling temporal dynamics of satellite observations in a recursive way. The accuracy assessment is evaluated based on 3,782 Landsat-based disturbance reference plots $(30 \mathrm{~m})$ from a probability sampling distributed throughout the Conterminous United States. Validation results show that the best F1 score of S-CCD is 0.793 with $20 \%$ omission error and $21 \%$ commission error, slightly higher than that of COLD (0.789). In addition, two disturbance sites respectively associated with fire and insect disturbances are used for qualitative map-based analysis. Both quantitative and qualitative analysis indicate that S-CCD can achieve noticeably less omission errors than COLD for detecting those disturbances with subtle/gradual spectral change such as insect attack and drought stress. S-CCD enables complete real-time monitoring, and up to $\sim 4.4$ times speedup for computation. This research addresses the need for near-real-time monitoring and large-scale mapping of forest health, and offers a new approach for operationally performing change detection tasks from long-term and dense Landsat-based time series.

\# This preprint has been submitted to Remote Sensing of Environment for peer review

*Corresponding author: Email remotesensingsuy@gmail.com Tele +1 5088264355

${ }^{1}$ Graduate School of Geography, Clark University, 950 Main Street, Worcester, MA 01610, USA

${ }^{2}$ Department of Natural Resources and the Environment, University of Connecticut, Storrs, CT 06269, USA

${ }^{3}$ Clark Labs, Clark University, Worcester, MA 01610, USA 
This preprint has been submitted to Remote Sensing of Environment for peer review

\section{Introduction}

In the last two decades, linked to the recent elevated air temperature and prolonged drought, an increase in the occurrence and severity of forest disturbance has been documented over large parts of the globe (Dale et al., 2001; Seidl et al., 2017; Turner et al., 1998); notably, insect outbreak (Kautz et al., 2017; Paritsis and Veblen, 2011),wildfire (Pechony and Shindell, 2010; Westerling, 2016) and drought (Allen et al., 2015). Forest disturbance events directly emit carbon to the atmosphere through oxidation and decomposition of wood (Masek et al., 2008), and yield significant impacts on ecosystem services of national forests such as climate regulation and biological diversity conservation (Curran and Trigg, 2006). Therefore, it is important to systematically gather information regarding the extent, timing and magnitude of forest disturbance in an accurate and timely manner, enabling an early warning and effective management to prevent further loss of forested land (Rogan and Mietkiewicz, 2015).

For decades, satellite remote sensing has been promoted as a key data source for operational forest monitoring (Pasquarella et al., 2017). Particularly, the opening of the Landsat archive (Woodcock et al., 2008) has led to improved opportunities for characterizing forest disturbances from a long-term and consistent Landsat time series (Zhu, 2017). Compared with low-resolution datasets such as Moderate Resolution Imaging Spectroradiometer (MODIS, 250-1000 m resolution), Landsat-based time series are provided in a sufficient temporal length of 40-year global record of fine-grained observations $(30 \mathrm{~m})$ (Masek et al., 2013). Therefore, Landsat is often perceived as the best free-access remotely sensed data source for resolving the full range of disturbance occurrence (Cohen et al., 2017; Cohen et al., 2016; Kennedy et al., 2014; Ye et al., 2018). Recently, the release of Landsat Analysis Ready Data (ARD) has eased automation for monitoring large-scale forest disturbances (Dwyer et al., 2018). The Landsat ARD gridded all available Landsat-4 and -5 TM, Landsat-7 ETM+ and Landsat-8 OLI/TIRS to an Albers Equal Area (AEA) Conic map projection, and are consistently geo-registered and atmospherically corrected; and hence holds the highest level of scientific standards and processing required for immediate use (Zhu, 2019). 
This preprint has been submitted to Remote Sensing of Environment for peer review

62 The Landsat ARD requires the minimum of user effort for data preprocessing, greatly facilitating a large-

63 scale and long-term time series analyses (Dwyer et al., 2018).

64 A wealth of methodologies on satellite-based time series analysis have been developed for land cover 65 change detection and characterization (Kennedy et al., 2010; Verbesselt et al., 2010a; Zhu and Woodcock,

66 2014b; Zhu et al., 2012). These algorithms are often categorized based upon their monitoring strategies:

67 offline or online monitoring (Bullock et al., 2019; Zhu, 2017). Offline monitoring focuses on a retrospective

68 analysis when the collection of time series data is completed, and seeks to reconstruct forest disturbance

69 history. The representative approaches for this category include LandTrendr (Kennedy et al., 2010), DBEST

70 (Jamali et al., 2015) and the ensemble approach (Bullock et al., 2019). Online monitoring is applied to a

71 practical scenario that the new observations are successively collected and processed in a timely fashion,

72 and hence can be used for near real-time monitoring. Representative approaches for online monitoring are

73 Breaks for Additive Season and Trend Monitor (BFAST Monitor) algorithm (Verbesselt et al., 2012) and

74 Continuous Change Detection and Classification (CCDC) (Zhu and Woodcock, 2014b; Zhu et al., 2019),

75 though they are also applicable for offline monitoring. The BFAST was originally designed for temporal

76 segmentation of MODIS-based time series (Verbesselt et al., 2010a), and was later modified (BFAST

77 Monitor) to address near real-time detection for drought-related vegetation disturbance (Verbesselt et al.,

78 2012). BFAST Monitor is a univariate approach that uses a single spectral band or index, while it has been

79 reported that multiple bands or indices is more preferable because forest disturbance has a multi-spectral

80 expression requiring multi-band inputs (Cohen et al., 2017; Zhu et al., 2019).

81 CCDC is a multivariate time-series model that uses all available Landsat 4-8 data for change

82 characterization and land cover classification (Zhu and Woodcock, 2014b). The CCDC first applies the

83 Fmask (Zhu and Woodcock, 2012) and Tmask algorithm (Zhu and Woodcock, 2014a) to screen clouds,

84 cloud shadows and snow, and then build a harmonic model for each spectral band based on remaining clear

85 observations. A breakpoint indicative of the timing of the disturbance is identified when the minimum

86 discrepancy between actual and predicted reflectance of spectral bands for a monitoring window is greater 
This preprint has been submitted to Remote Sensing of Environment for peer review

87 than a predefined change threshold (Zhu and Woodcock, 2014b). Recently, an improved algorithm called

88 'COntinuous Monitoring of Land Disturbance'(COLD) was developed based upon CCDC (Zhu et al., 2019).

89 COLD introduced several improvements such as disturbance extraction, temporally-adjusted Root Mean

90 Square Error (RMSE), change angles for disturbance confirmation (Zhu et al., 2019). An important finding

91 for COLD is that using the highest frequency for harmonic model updates, that is per observation instead

92 of per a time span for the CCDC, can reduce $20 \%$ commission errors (Zhu et al., 2019). Zhu et al. (2019)

93 tested the performance of COLD using 7,200 Landsat time series plots randomly selected across the

94 conterminous United States, and reported that the COLD algorithm achieves a higher accuracy than CCDC,

95 with $27 \%$ omission error and a $28 \%$ commission error for a variety of land disturbance types.

96 However, while the COLD algorithm has achieved improved performance for change detection accuracy,

97 there are several issues limiting its implementation for operational monitoring. First, COLD is

98 computationally expensive, which takes $\sim 5000$ computing hours for a Landsat ARD time series (Zhu et al.,

99 2019). The high computational requirement imposes limits on the application of COLD for a wide range of

100 scientific research, especially for a large-area mapping such as at a state or continental scale. The cause for

101 the slow speed is mainly from the per observation updating approach (Zhu et al., 2019). For example, if the

102 time series of a single pixel has 500 clear observations, COLD needs to re-train the model using the 'Least

103 Absolute Shrinkage and Selection Operator' (LASSO) algorithm for approximately 500 times to complete

104 a detection for this pixel. Computational redundancies arise from reconstructing models from scratch for

105 each new observation being added to the time series. Second, the former version of COLD, namely CCDC,

106 was designed to detect land cover change, and is less helpful for detecting those disturbances that yield

107 small to medium spectral change magnitude (Brown et al., 2019; Cohen et al., 2017; Zhu et al., 2019). A

108 possible reason provided is that CCDC identifies gradual changes as the slope in the harmonic regression

109 model as opposed to attributing them to a change on a specific date. Though this issue was alleviated by

110 COLD, it has not been fully solved yet because COLD reported only $60 \%$ producer accuracy for the

111 disturbance category 'stress' (Zhu et al., 2019). Third, within the current workflow of COLD, near real- 
This preprint has been submitted to Remote Sensing of Environment for peer review

112 time monitoring is not fully operational because: 1) COLD is a memory-intensive algorithm which requires

113 loading all images to update model coefficients and to calculate temporally-adjusted RMSE for each new

114 observation; 2) a certain steps of COLD such as minimum RMSE require an input of a complete time series,

115 which does not satisfy the need of near real-time monitoring.

116 In this study, we describe a new algorithm called 'Stochastic Continuous Change Detection' (S-CCD) that

117 is developed to detect forest disturbance from Landsat time series in a recursive fashion. S-CCD introduces

118 the state space theory into the current framework of COLD, aiming to address three objectives: 1) to

119 enhance detection accuracy, especially for those forest change with small spectral change magnitudes while

120 keeping a low rate for commission errors; and 2) to provide an operational framework for near real-time

121 monitoring; 3) to improve computational efficiency, enabling a long-term time series analysis for a large-

122 area forest disturbance characterization.

123 In what follows, we first provide an intuitive explanation for state space models and the Kalman filter as

124 the mathematical foundation of S-CCD (Section 2), describe our S-CCD algorithm focusing on different

125 steps with COLD (Section 3), introduce our reference dataset and validation metrics (Section 4) and exhibit

126 the results for both quantitative and qualitative evaluation (Section 5), and finally discuss advantages and

127 future work for S-CCD (Section 6). Of particular note is that Section 5.3 presents a high-performance

128 software package for both S-CCD and COLD implemented in C language.

\section{State space model and the Kalman filter}

130 The new approach built upon the State Space Model (SSM), an established time-series mathematical

131 framework that allows for modeling a dynamic of observed measurements as being explained by a vector

132 of latent state variables. The SSM has two foundational 'stochastic' assumptions: 1) observations are

133 formulated as a sum of a stochastic item linked to the uncertainty within the data themselves, namely

134 'observational noise' $\left(\epsilon_{t}\right)$, and a vector of latent variables called 'states' $\left.\left(\alpha_{t}\right) ; 2\right)$ states which are evolving

135 over time as a stochastic process with being affected by a 'process noise' $\left(\eta_{t}\right)$. Different from classical 
This preprint has been submitted to Remote Sensing of Environment for peer review

decomposition models such as harmonic regression used in BFAST and CCDC, SSM allows for trend and

'Stochastic Continuous Change Detection'.
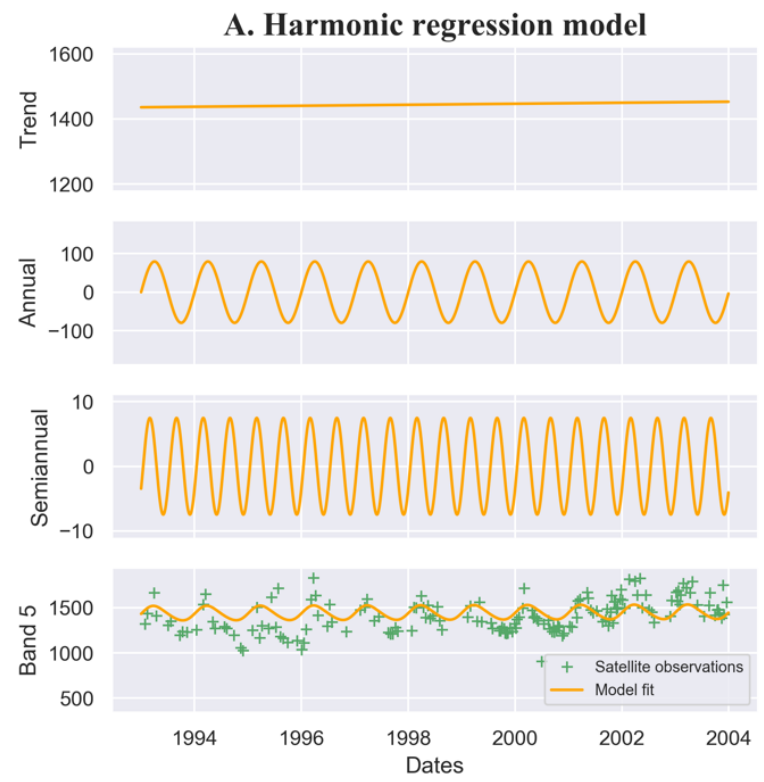

Observation equation: $y_{t}=Z a_{t}+\epsilon_{t}, \epsilon_{t} \sim \mathrm{N}(0, \mathrm{H})$

State equation: $a_{t+1}=T a_{t}+\eta_{t}, \eta_{t} \sim \mathrm{N}(0, \mathrm{Q})$
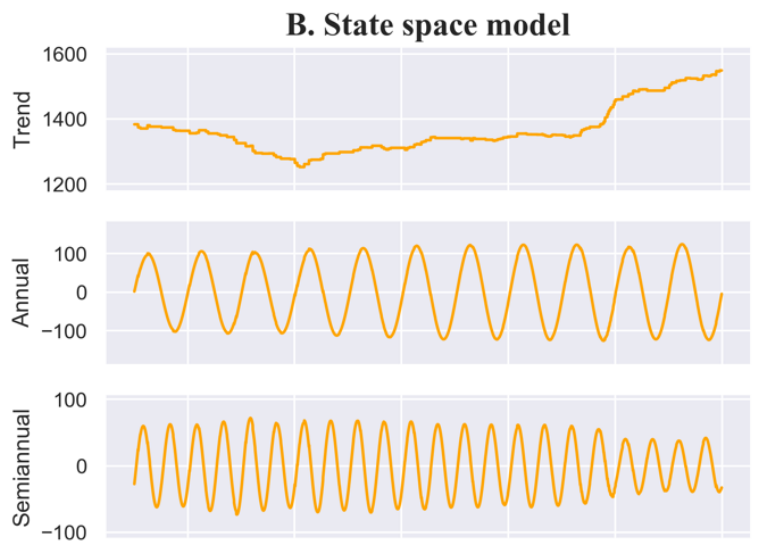

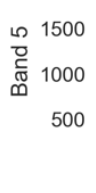

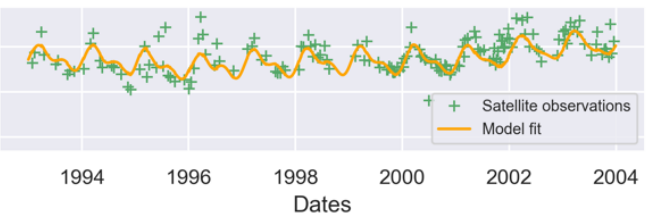

Fig. 1. The comparison between a harmonic regression model (A) and a state space model (B) for fitting curves. The harmonic regression model has a rigidity for consistent coefficients such as intercepts, slope and Fourier coefficients, while the state space model assumes that each component is evolving as a stochastic process and yields the optimal estimate for each time step based upon its state covariance and observational noise, so that the model coefficients vary over time.

The general Gaussian SSM can be written in the form as below (Durbin and Koopman, 2012):

Where $y_{t}$ is the observation at time $t, \epsilon_{t}$ and $\eta_{t}$ are two mutually independent random variables that follow a normal distribution with mean 0 and variance $H$, and variance $Q$, respectively. $Z$ is a system matrix in a binary form, which indicates those state items that directly contributes to the observation. $T$ is a transformation matrix defining how a state vector evolves over time (mathematical definitions for $Q, Z$ and 
This preprint has been submitted to Remote Sensing of Environment for peer review

154 series models, owing to its ability of allowing for structural components to be modeled explicitly by state

155 variables (Brockwell and Davis, 2013; Durbin and Koopman, 2012). SSM holds great promise for 156 processing remote sensing time series, which are well known for the structure of 'trend + cycles' (Eastman 157 et al., 2013; Verbesselt et al., 2010b; Zhu and Woodcock, 2014b).

158 The SSM for the state of 'trend' is formulated as a random-walk model:

$\mu_{t+1}=\mu_{t}+\xi, \xi \sim \mathrm{N}\left(0, \sigma_{\xi}^{2}\right)$

160 Where $\xi$ is a process noise item for the trend. The 'cycle' process requires two state variables to define:

$c_{t+1}=c_{t} \cos \lambda_{c}+c_{t}^{*} \sin \lambda_{c}+\omega_{t}, \omega_{t} \sim \mathrm{N}\left(0, \sigma_{\omega}^{2}\right)$

162

$c_{t+1}^{*}=-c_{t} \sin \lambda_{c}+c_{t}^{*} \cos \lambda_{c}+\omega_{t}^{*}, \omega_{t}^{*} \sim \mathrm{N}\left(0, \sigma_{\omega^{*}}^{2}\right)$

163 Where $\lambda_{c}$ is the frequency of the cycle, $\omega_{t}$ and $\omega_{t}^{*}$ are independent noise items. $c_{t}$ is the primary cycle

164 state, while $c_{t+1}^{*}$ is an accessory state variable that is not included for prediction of $y_{t}$ and only used to

165 enable a recursive form of mathematical computation (so its corresponding element in $Z$ is 0 ). For a classic

166 model for 'trend + annual cycle + semi-annual cycle', the state vector can therefore be given by

$167 a_{t}=\left(\mu_{t}, c_{t, \text { annual }}, c_{t, \text { annual }}^{*}, c_{t, \text { semi }}, c_{t, \text { semi }}^{*}\right)^{\prime}$

168 Compared with 6-coefficients harmonic model, our state space model has no component corresponding to

169 'slope', because S-CCD is essentially a Markov model forecasting present states based on only the last

170 observation, not all historical observations (i.e. decreasing or increasing trend). The advantages for

171 eliminating 'slope' component will be discussed in Section 6.

172 The Kalman filter is the most common tool providing an operational treatment for SSM. The Kalman filter

173 was first developed for estimating real-time trajectory of the spacecraft for the Apollo program (Schmidt,

174 1981), and was later introduced to other application domains such as the control of linear systems (Davis

175 and Vinter, 1985) and econometric modeling (Pasricha, 2006). Recently, the Kalman filter was applied to

176 improve satellite-based time series analysis for applications such as crop phenology estimation (Vicente- 
This preprint has been submitted to Remote Sensing of Environment for peer review

177 Guijalba et al., 2014), synthetic NDVI image generation (Sedano et al., 2014) and near real-time monitoring 178 of defoliation (Olsson et al., 2016). The Kalman filter is claimed to produce an optimal estimate in the sense 179 that it always reaches the minimum mean square error, and is capable of predicting measurements in a 180 recursive manner so that new measurements can be processed as they arrive (Durbin and Koopman, 2012; 181 Kalman, 1960). As such, the Kalman filter has great potential for being a fundamental tool of satellite-based 182 near real-time monitoring.

183 Fig. 2 presents an intuitive explanation of a Kalman filter for adjusting its modelling curve successively 184 once a new observation is available. The Kalman Filter defines one-ahead-step prediction for the date $t$ as

$185 \widehat{y_{t}}=a_{t} * Z$

186 When a new observation, '2000-12-27', is introduced into the time series, there is a discrepancy between

187 the one-ahead-step prediction (the solid orange dot) and the new observation (the green cross) called

188 'innovation'. In a Kalman filter, the 'innovation' can be divided into two components: 1) observational

189 noise; and 2) model updates brought by the new observation. The Kalman gain is the relative weight 190 assigned to actual model updates, which can be estimated by covariances of states and observational noise.

191 Once the Kalman gain is computed, the Kalman filter can filter out observational noise from the innovation.

192 The new model coefficient will be adjusted based upon the remaining part that is considered only associated

193 with actual model updates, yielding a new fitting curve 'filtered state' (the blue dot). The filtered states (or 194 adjusted coefficients) will then be used to predict the next observation (the dashed orange line). 


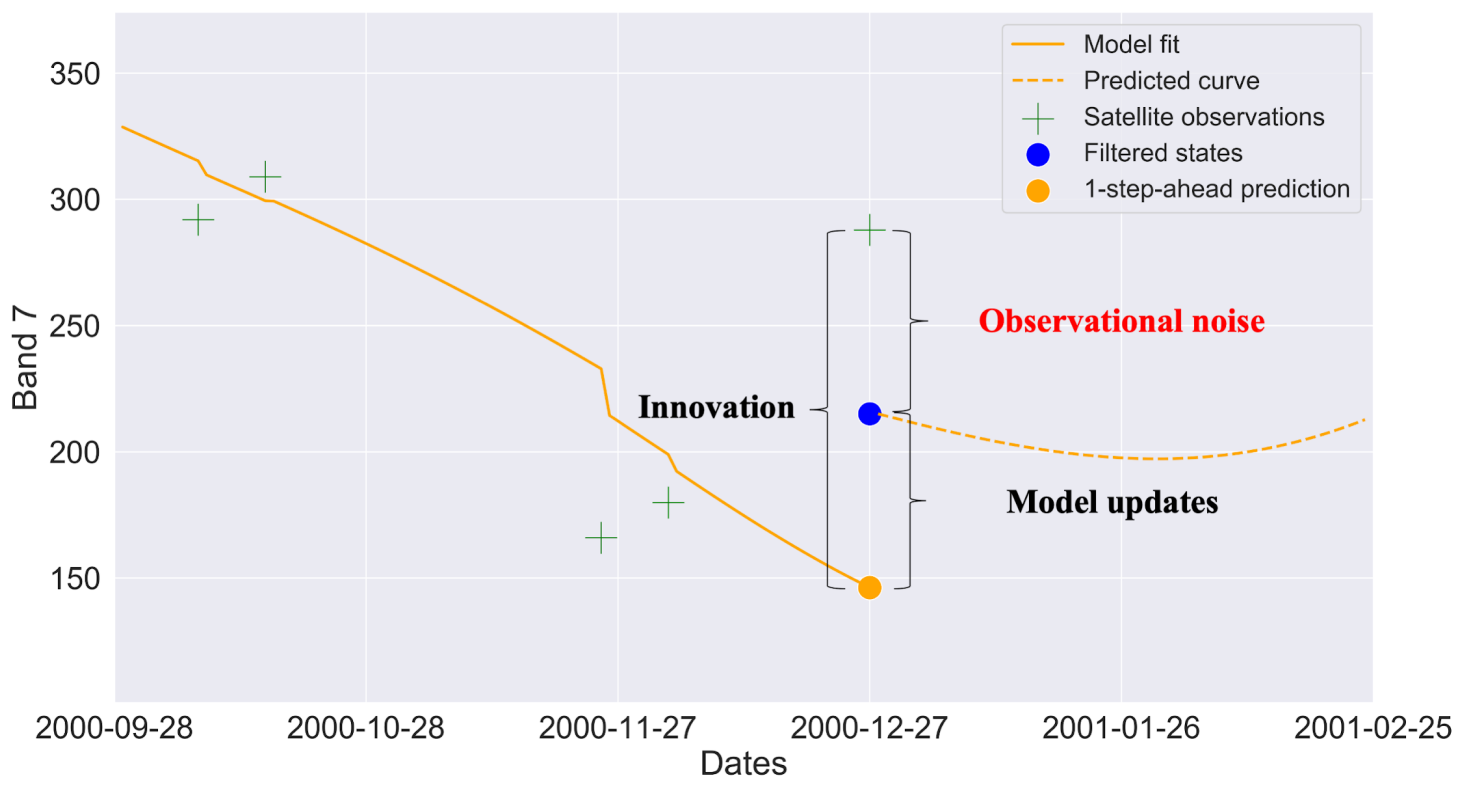

Fig. 2. An intuitive explanation of Kalman filter for a Landsat time series. When a new observation at the date of ' $2000-12-27$ ' is introduced to the system, the Kalman filter measures the difference between the actual observation (the green cross at '2000-12-27') and the predicted value (the green dot), namely 'innovation'. The fitting curve for the model will be adjusted to align with the optimal estimate for the current states (the 'blue dot') by filtering out 'observational noise' from 'innovation'.

Vegetation dynamics often exhibit a complex trend, which is not guaranteed to be adequately approximated by a single linear mode (Burkett et al., 2005; Zhao et al., 2019). The assumption for stochastically varying states for SSM and Kalman filters avoids the rigidity of classical decomposition that assumes the stationarity of linearity and seasonality, hence complex dynamics from time-series data are uncovered and more local fluctuations can be captured (Brockwell and Davis, 2013). The flexibility of dynamic modeling directly leads to better fitness of the model, and hence potentially increases the sensitivity of the model to subtle changes because change magnitudes are often calculated relative to measurement of model fitness such as Root Mean Square Error (RMSE). Other advantages of a state space analysis for satellite-based time series include: 1) simple mathematical treatment of missing data (shown in Appendix) which is critical for dealing with satellite-based time series known for its temporal irregularity; 2) explicit consideration for 
This preprint has been submitted to Remote Sensing of Environment for peer review

213 measurement uncertainties for the noisy nature of remote sensing data; 3) high computational efficiency 214 due to its recursive form.

\section{3. Method}

216 Fig. 3 presents the workflow of S-CCD. S-CCD consisted of three primary stages: data preparation, model 217 initialization and continuous monitoring. We used five spectral bands of surface reflectance products (green, 218 red, NIR, SWIR1 and SWIR2) as the algorithm inputs because Zhu et al. (2019) have shown that these five 219 spectral inputs alone can achieve the best performance compared with being combined with vegetation 220 indices. We applied the same steps as COLD for S-CCD for data preparation, for which we refer to Section 2213.1 .1 of (Zhu et al., 2019). 
This preprint has been submitted to Remote Sensing of Environment for peer review

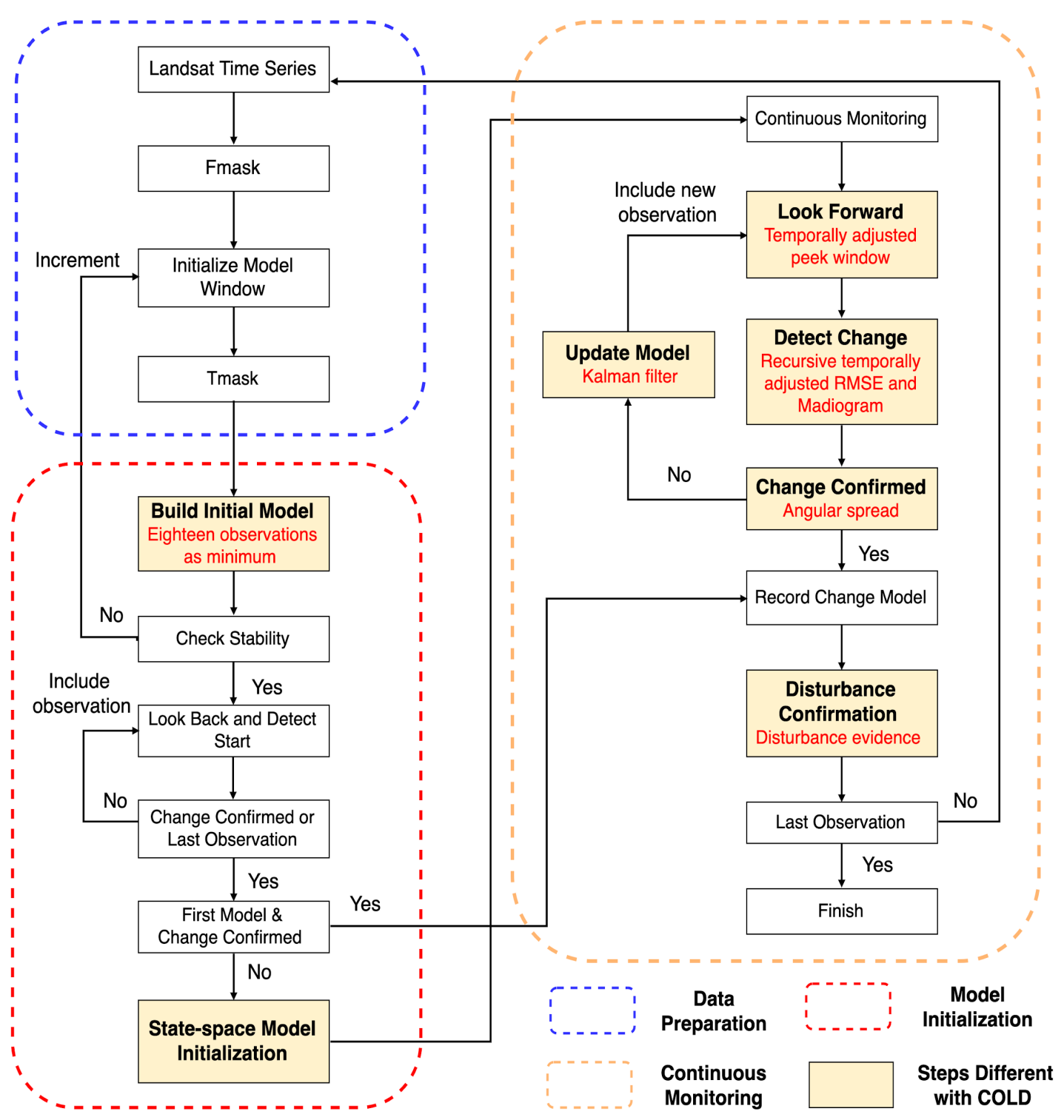

Fig. 3. The workflow of the proposed Stochastic Continuous Change Detection (S-CCD). Same as the original COntinuous monitoring of Land Disturbance (COLD), the workflow consists of three stages: 1) data preparation; 2) model initialization; 3) continuous monitoring. The different steps between S-CCD and COLD are highlighted as yellow polygons.

\subsection{Build Initial Model}

After a clear time series is prepared by Fmask (Zhu and Woodcock, 2012), initialization model window and Tmask (Zhu and Woodcock, 2014a), S-CCD needs to seek a stable stage to define a statistical reference for change identification which adopts the stability test and 'looking back' procedure (Zhu et al., 2019). 
This preprint has been submitted to Remote Sensing of Environment for peer review

231 The subtle modification for S-CCD is that we used 18 instead of 12 as the required minimum number of

232 clear observations for an initialization window: our new approach assumes a fixed structure of "trend +

233 annual + semiannual' for a time series which can be equivalent to 6-coefficients harmonic model. The

234 suggested minimum observation window for a LASSO regression is 'number of coefficients * 3', hence the

235 minimum observation number is set as 18 . The reason for excluding a trimodal component (8-coefficient

236 model) is discussed in Section 6.

\section{$237 \quad 3.2$ State space model (SSM) initialization}

238 In S-CCD, an additional step is to initialize the parameters and the structural elements for state space models

239 before the continuous monitoring starts. The initial SSM parameters include observational noise $(H)$,

240 process noise $(Q)$, initial states $\left(a_{0}\right)$ and initial covariance $\left(P_{0}\right)$.

241 The parameters $H$ and $Q$ are the two most important SSM parameters, representing the uncertainty level for

242 observations and each stochastic process. They are often estimated by maximizing likelihood through a

243 Quasi-Newton numerical searching algorithm in literature (Durbin and Koopman, 2012; Helske, 2016).

244 However, after initial tests, we learned that the Quasi-Newton algorithm was extremely inefficient for

245 processing millions of pixel-based time series. To overcome the issue, we designed a fast method for

246 estimating $H$ and $Q$. We set the observation noise as the squared RMSE of LASSO regression for the

247 initialization window: $H=R M S E * R M S E$. To estimate $Q$, the Kalman filter with the initial process noise

$248 Q_{\text {ini }}=\operatorname{diag}(0,0,0,0,0)$ is first applied to estimate filtered states for each observation within the

249 initialization window. Then, the process noise is corrected using the results of estimated filtered state from

250 the first run: the filtered trend state is ideally consistent if there is no process noise, and thereby, a single

251 process noise can be estimated as:

252

$\xi_{n}=\left(\mu_{t(n) \mid t(n)}-\mu_{t(n-1) \mid t(n-l)}\right) / \operatorname{sqrt}(t(n)-t(n-1))$

253 Where $t(n-1)$ and $t(n)$ is the date for two temporally neighboring observations, namely $t-1$ th and $t$ th

254 observation, $\mu_{t(n-l) \mid t(n-l)}$ and $\mu_{t(n) \mid t(n)}$ represents filtered trend states for these two observations. 
This preprint has been submitted to Remote Sensing of Environment for peer review

255 The process noise for the trend state can be calculated as $\sigma_{\xi}^{2}=\operatorname{var}\left(\xi_{n}\right)$. The process noises for components

256 are viewed as being proportional to the mean of the absolute value of the corresponding states, so the

257 seasonal process noise can be calculated as:

$258 \quad \sigma_{\omega}^{2}=\frac{\sum_{n=1}^{N}\left|C_{t(n)}\right|}{\sum_{n=1}^{N}\left|\mu_{t(n)}\right|} \sigma_{\xi}^{2}$

259 Initial states $a_{0}$ are estimated from using 6-coefficient LASSO regression to fit all observations within an

260 initialization window. Initial covariance $P_{0}$ represents the uncertainty level of $a_{0} . P_{0}$ is often assumed to be

261 the isotropic matrix, $P_{0}=\lambda \mathrm{I}$. The literature indicates that the uncertainty of Landsat surface reflectance

262 product is under the surface reflectance $(\mathrm{SR})$ specification, defined as $5 \% \times \mathrm{SR}+0.005$ (Claverie et al., 263 2015; Ju et al., 2012). Accordingly, we used 5\% for estimating uncertainties of initial states, that is $\lambda=$ $264\left(Z \alpha_{t} * 5 \%\right)^{2} / \operatorname{sum}(Z)$.

\section{$265 \quad 3.3$ Temporally adjusted peek window}

266 For each newly-collected clear observation, S-CCD compares predictions from the Kalman filter and actual 267 observations based upon a number of consecutive anomaly observations, called the 'peek window' for 268 CCDC-like approaches (Davis et al., 2017). COLD approach determines the number of consecutive 269 observations via calculating the median revisit days across the entire time series. If the median revisit days 270 are shorter than normal Landsat temporal density (16 days), more consecutive observations need to be

271 included, and the probability threshold that all observations need to exceed is decreased to compensate (Zhu

272 et al., 2019). This approach requires an entire time series to compute median revisit days. We developed a

273 completely online approach for adjusting the peek window for S-CCD. The number of observations for an

274 temporally-adjusted peek window is defined as the minimum number satisfying 1) minimum requirements

275 for consecutive observations, i.e., conse $_{d e f}$; and 2) the minimum peek days (min_peek_days):

276 conse $_{\text {def }}=\min \left\{x \mid x \geq\right.$ conse $_{\text {def }}$ and $\operatorname{span}(x) \geq \min \_$peek_days $\}$ 
This preprint has been submitted to Remote Sensing of Environment for peer review

277 The defaulted consecutive number conse $_{d e f}=6$. The min_peek_days is related to the temporal span

278 indicating consistency of a disturbance event affecting vegetation. If min_peek_days is set to be too long,

279 signals of a disturbance might be diluted due to post-disturbance forest regrowth; if too short, some 280 ephemeral changes such as soil moisture change might be misidentified as disturbance. As the normal 281 Landsat density is 16 days per clear observation (Zhu et al., 2019), the normal peek window width for 282 min_peek_days is $6 * 16=96$ days. We explored the algorithm performance by using several 283 min_peek_daysbelow the normal peek window width (e.g. 60, 70, 80 days). Choosing min_peek_daysas

28480 days shows the best result (see S1 in the supplementary material). The threshold probability is also 285 adjusted when a peek window chooses a consecutive observation number larger than conse $e_{\text {def }}$, following 286 Equation S4 in (Zhu et al., 2019).

287 Another advantage of the new peek window in terms of COLD approach is making it feasible to account 288 for variability in Landsat observation frequency across not just space, but also time. The new definition of 289 the peek window considers that the consecutive number is not fixed for a single time series. Time series 290 segments collected in the early days (e.g. before the launch of Landsat 7 ETM+ on 1999) often have a lower 291 temporal density than the most recent collections. Large changes in frequency driven by the number of 292 active sensors aboard Landsat satellites had an influence on the CCDC/COLD change detection records 293 (Brown et al., 2019): higher detection rates often occur at higher observation frequency, including 294 commission errors brought by ephemeral forest change. The new peek window enables an adjustment of 295 consecutive observations based upon the local temporal density for the peek window, because it defines the 296 peek window according to the physical attribute of a disturbance signal, namely lasting days, not satellite 297 observation count.

\section{3.4 Recursive temporally-adjusted RMSE}

299 Squared differences between predictions and observations is used to evaluate deviation of the current peek 300 window from the 'stable stage'. RMSE is used to normalize the square difference, which is critical for 
This preprint has been submitted to Remote Sensing of Environment for peer review

301 decisions on the occurrence of breakpoints. Considering that RMSE often exhibits a yearly pattern over the 302 whole time series (Zhu et al., 2015), the COLD algorithm employs a temporally-adjusted RMSE that is 303 calculated based on the temporally closest 24 observations to the peek window. For operational near real304 time monitoring, this temporally-adjusted RMSE needs to be re-computed by loading all images back into 305 the model for a new observation with its date. We designed a novel recursive method based upon two 306 histograms respectively for clear observation counts (Fig. 4A) and square of RMSE (Fig. 4B) for Days of 307 Year (DOY), which eliminates the need for processing all images for each new observation. Both 308 histograms are defined as a bin width of 6 days, and 61 bins in total. The two histograms keep updating 309 when a new observation is available. To compute a temporally-adjust RMSE for a new observation, S310 CCD will start from the bin at the middle date of the peek window ('Step 1' in Fig. 4A), and expand the 311 RMSE window by an increment of one bin on the left and the right side each time, until the RMSE window 312 include $\geq 24$ observations ('Step 3' in Fig. 4A). The temporal RMSE is computed as the average 313 RMSE*RMSE based on the sum of RMSE*RMSE and the total count within the resultant RMSE window. 

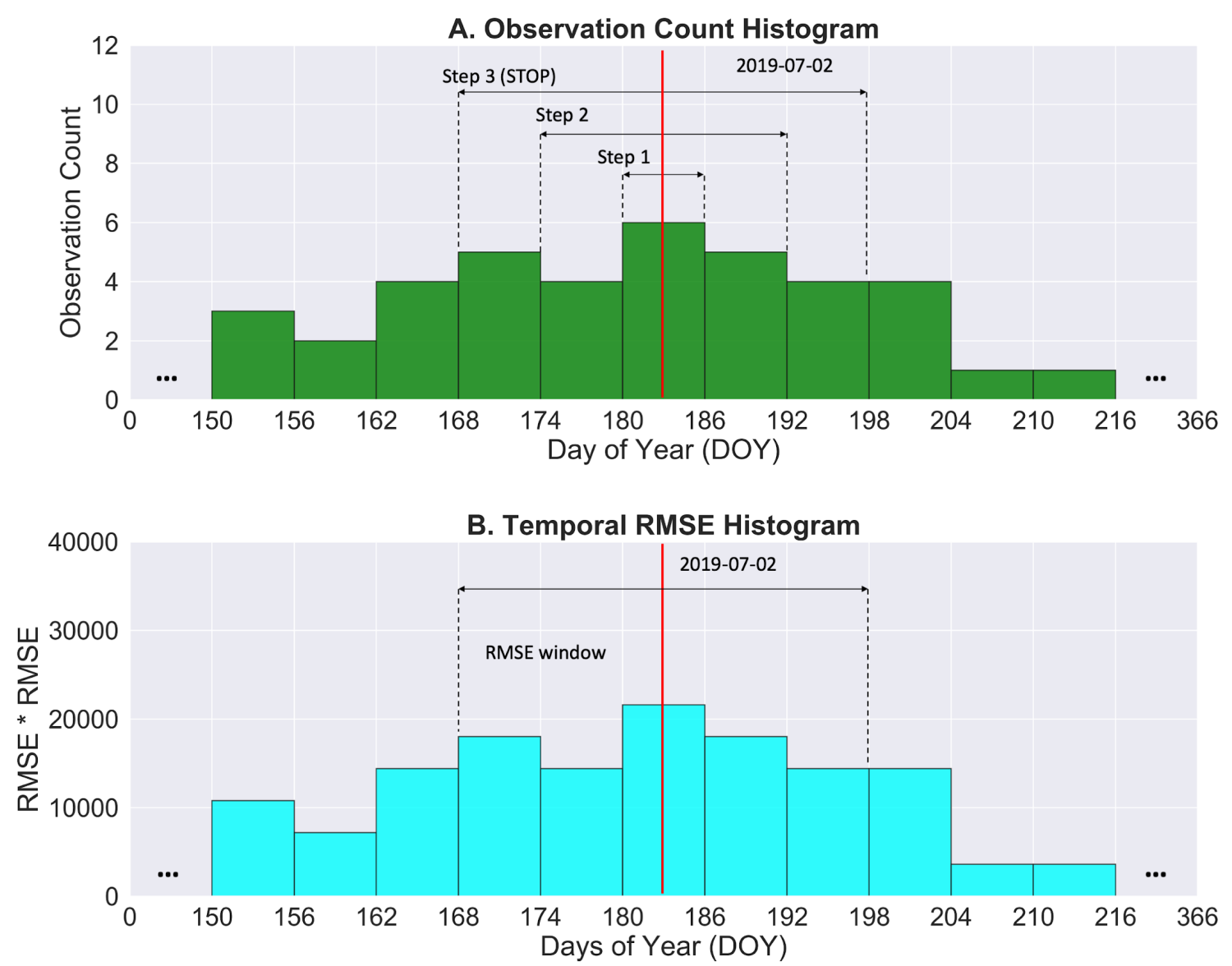

Fig. 4. The explanation for a recursive calculation of temporal RMSE: the algorithm searches a RMSE window that just covers $\geq 24$ observations by an increment of a bin on both sides in an observational count histogram (Fig. 4A). Once it stops (Step3 in Fig. 4A), the temporal RMSE is computed as the average RMSE* RMSE within the resulting RMSE window.

320 Likewise, to enable near real-time monitoring, we used a dynamic minimum RMSE (or temporal lag-1

321 madogram), instead of a static minimum RMSE used in (Zhu et al., 2019), to define the minimum value of

322 RMSE for each band: the madogram is updated each year using all clear past observations.

323 Therefore, the new RMSE for S-CCD is computed as

$324 R M S E_{i}=\max \left(\sqrt{\frac{\sum_{\in \varphi} R M S E_{i, b} * R M S E_{i, b}}{\sum_{\in \varphi} \text { count }_{i, b}}}\right.$, dynamic_min_RMSE $\left.E_{i}\right)$

325 Where $\varphi$ is a group of all bins (b) within the resultant RMSE window, and $i$ is $i$ th Landsat band. 
This preprint has been submitted to Remote Sensing of Environment for peer review

326 We define the Standardized Change Vector $\left(S C V_{n}\right)$ as a vector of the difference between a multispectral

327 observation $n$ at time $t(n)$ and its one-step-ahead prediction relative to $R M S E$ :

328

$S C V_{n}=\left[\frac{y_{t(n), l}-\hat{y}_{t(n), I}}{R M S E_{1}}, \frac{y_{t(n), 2}-\hat{y}_{t(n), 2}}{R M S E_{2}}, \cdots \frac{y_{t(n), B}-\hat{y}_{t(n), B}}{R M S E_{B}}\right]$

$329 B$ is the number of bands used, i.e., 5 (green, red, NIR, SWIR1 and SWIR2). Similar to the COLD algorithm,

330 we used the minimum change magnitude for each change vector within the peek window to detect the

331 breakpoint. The minimum change magnitude $(M C M)$ is used as the indicator of breakpoints, which is

332 calculated as the minimum norm of $S C V_{n}$ for a peek window. MCM follows the Chi-squared distribution

333 with $k$ degree of freedom (Zhu et al., 2019), where $k$ is the number of the used spectral bands, namely ' 5 '.

334 A breakpoint candidate is identified if it meets the condition, where $p$ refers to the current peek window:

$335 M C M=\left\{\left\|S C V_{n}\right\|^{2}\right\} \sim \chi^{2}(k)>\chi_{\text {threshold }}^{2}(5)$

\subsection{Confirm change using angular spread}

337 Ephemeral and systematic noise may also lead to relatively large change magnitudes for all observations

338 within a peek window. The disturbance signal, however, should have a consistent change direction for

339 multispectral surface reflectance bands. Therefore, the time series model needs to confirm the change from

340 the consistency of change angles once the Chi-squared distribution test is passed. COLD confirms breaks

341 using the mean included angle between pairs of neighboring change vectors smaller than 45 degrees. We

342 found that this strategy is sensitive to outliers: for example, if there is an outlier in the six consecutive

343 observations, two out of five neighbor pairs will be affected by this outlier ( $40 \%$ of the candidate angles);

344 if there are two outliers in a peek window, more than half of neighbor pairs are biased. We designed a new

345 change angle index called 'angular spread', referred as the angle between the standardized change vector

346 for $n$th observation $\left(S C V_{n}\right)$ and the median standardized change vector (MedSCV) (see Fig. 5). The mediam

347 change vector of a peek window here is used to represent the average of the spectral response of a

348 disturbance. The observations for a disturbance should concentrate around the median change vector in an 
This preprint has been submitted to Remote Sensing of Environment for peer review

ideal condition. We define 'Mean Angular Spread' for a peek window (Equation 14) to represent the average angular departure of each candidate change vector to medium change vector. We compared the

351 performance of the maximum mean included angle as 45 degrees (the COLD approach) and the maximum

352 angular spread with 30, 45 degree. The best result is achieved by using angular spread as 30 degrees (Fig.

353 S2 in the supplementary material). The advantage of using 'Mean Angular Spread' is that is less sensitive

354 to outliers existing in a peek window. For example, an outlier only affects a single change angle out of six

355 angles for a default peek window (16.7\% of the candidate angles).

356

Mean Angular Spread $=\frac{1}{\text { conse }_{\text {adj }}} \sum_{n=1}^{\text {conse }}{ }^{\text {adj }} \theta_{\left(S C V_{n}, \text { MedSCV }\right)}<30^{\circ}$

B2

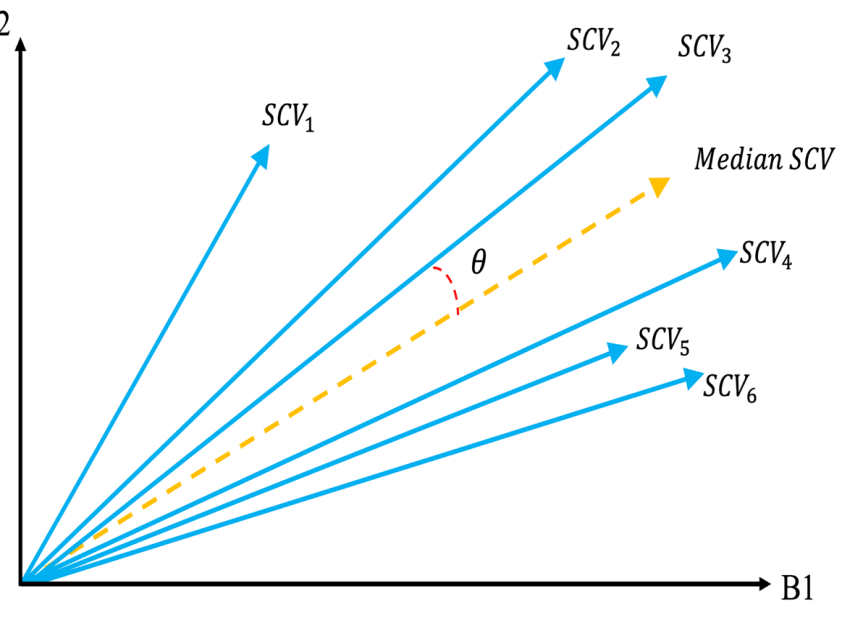

Fig. 5. The explanation of 'angular spread' for a peek window consisted of 6 consecutive observations with two spectral bands $(B 1, B 2)$. Angular spread is computed as the mean angle for $\theta$, which is the included angle between the Standardized Change Vector $\left(S C V_{1}, \ldots, S C V_{6}\right)$ and the Median Standardized Change Vector (Medium SCV).

\subsection{Update model using the Kalman filter}

364 For those observations that are identified as being unchanged, non-consecutive outlier removal will be used 365 with a change probability of 0.99999 (same as Zhu et al. (2019)). If the observation passes the outlier test, 366 it will be inputted to the system to update the model using the Kalman filter (see the Appendix). Instead of 
This preprint has been submitted to Remote Sensing of Environment for peer review

rebuilding LASSO regression each time, the Kalman filter has an extremely simple mathematical treatment

368 for updating models.

369

370

371

372

373

374

375

376

377

378

379

380

381

382

383

384

385

386

387

388

389

390

\subsection{Disturbance identification}

Breakpoints detected by the above procedure are not necessarily associated with forest disturbance, but maybe forest recovery. As the final step, we need to single out those breaks that are related to forest health decline led by disturbance. A typical forest disturbance will cause lower NIR, higher Red and higher SWIR values. Such spectral change, however, might be asynchronous. For example, when a forest is attacked by mountain pine beetle, the increase of SWIR often occurs first due to increased water stress, then is increasing red band, and finally the NIR band decreases owing to needle drop. Hence, we created an index called 'disturbance evidence' (see Equation 15) based on the medium Standardized Change Vector (MedSCV) associated with detected breakpoints. 'Disturbance evidence' aims to provide a combined analysis for multiple bands instead of a single band index. The breaks that are identified as being disturbance-related need to have a disturbance evidence larger than zero:

Disturbance evidence $=M e d S C V_{R E D}-M e d S C V_{N I R}+M e d S C V_{S W I R 1}>0$

We compared 'Disturbance Evidence' with COLD disturbance extraction with thresholds 0, -0.01, -0.02, and -0.03. 'Disturbance Evidence' had a higher best F1 score better than all other COLD disturbance extraction methods at all five thresholds (see Fig. S3 in the supplementary material).

\section{Study area, data and accuracy assessment}

Our accuracy assessment is consisted of a quantitative (plot-based) analysis against a comprehensive forest disturbance dataset, and qualitative (map-based) evaluation over two disturbance sites. For the quantitative accuracy assessment, a benchmark forest disturbance database (Cohen et al., 2016; Zhu et al., 2019) is chosen that includes 3,503 Landsat-based forest plots across the conterminous United States (US) with well-interpreted disturbance timing and types. The plots were sampled by a two-stage stratified cluster design (Cohen et al., 2016). A stratified sampling was first applied to select 180 Thiessen Scene Areas 
This preprint has been submitted to Remote Sensing of Environment for peer review

(TSAs; (Kennedy et al., 2010)) out of 420 TSAs divided into Mountain West, Lowland West, Central,

392 Northeast and Southeast US ecoregions as strata. For the second stage of sampling, 7,200 initial Landsat

393 plots were randomly chosen with a sample of 40 Landsat plots for each TSA, and then those plots with the

394 primary type of its land cover type was 'forest' at any time during the period of 1984-2012 were selected,

395 following the procedure of Cohen et al. (2016). Further, reference plots that do not satisfy the criteria of

396 enough clear sky observations (at least 24), have large data gaps (more than three years without any

397 observations), or have a disturbance with an interval of longer than 10 year, or difficult to interpret were

398 excluded (Zhu et al., 2019). As a result, a total of 3,782 forest plots across the conterminous US were

399 identified as our reference samples.

400 For the Landsat plots, forest disturbance occurrence between 1984 and 2012 were interpreted by USDA

401 Forest Service by combining plot-based time series visualization and high resolution Google Earth images

402 (Cohen et al., 2016); these samples were checked and corrected by five interpreters by eliminating the

403 disturbance plots that were not visually confirmed from the Landsat time series (Zhu et al., 2019). Causal

404 agent class of disturbances were interpreted with the aid of high-resolution Google Earth imagery, and an

405 ancillary database from multiple government agencies such as U.S. Forest Service Aerial Detection

406 Surveying maps ${ }^{4}$, Natural Resource Manager (NRM) database ${ }^{5}$, etc (Zhu et al., 2019). As a result, 3,782

407 forest plots were separated into 2,704 undisturbed plots and 1,078 disturbed plots, which have 1,413

408 disturbance occurrences in total (some plots have successive disturbance occurrences such as fire and

409 timber harvest). Among these disturbance occurrence, the most causal agent of disturbances is harvest $(\mathrm{n}=$

410 903), followed by mechanical $(n=149)$, stress $(n=141)$, fire $(n=127)$, and others $(n=83$, e.g., hydrology,

411 wind, debris, land use change). For a detailed description of these disturbance causal agent classes, we refer

412 to (Cohen et al., 2016). The spatial distribution of 3,782 Landsat plots labeled as undisturbed or the number

413 of disturbance occurrences is shown in Fig. 6. We randomly selected 50\% of the reference samples $(1,891$

\footnotetext{
${ }^{4}$ http://www.foresthealth.fs.usda.gov/portal/Flex/IDS

5 https://www.fs.fed.us/nrm/index.shtml
} 
414 Landsat plots) for algorithm development and parameter calibration. The other $50 \%$ of the reference

415 samples were used as a holdout validation set to evaluate the comparative performance of S-CCD and 416 COLD.
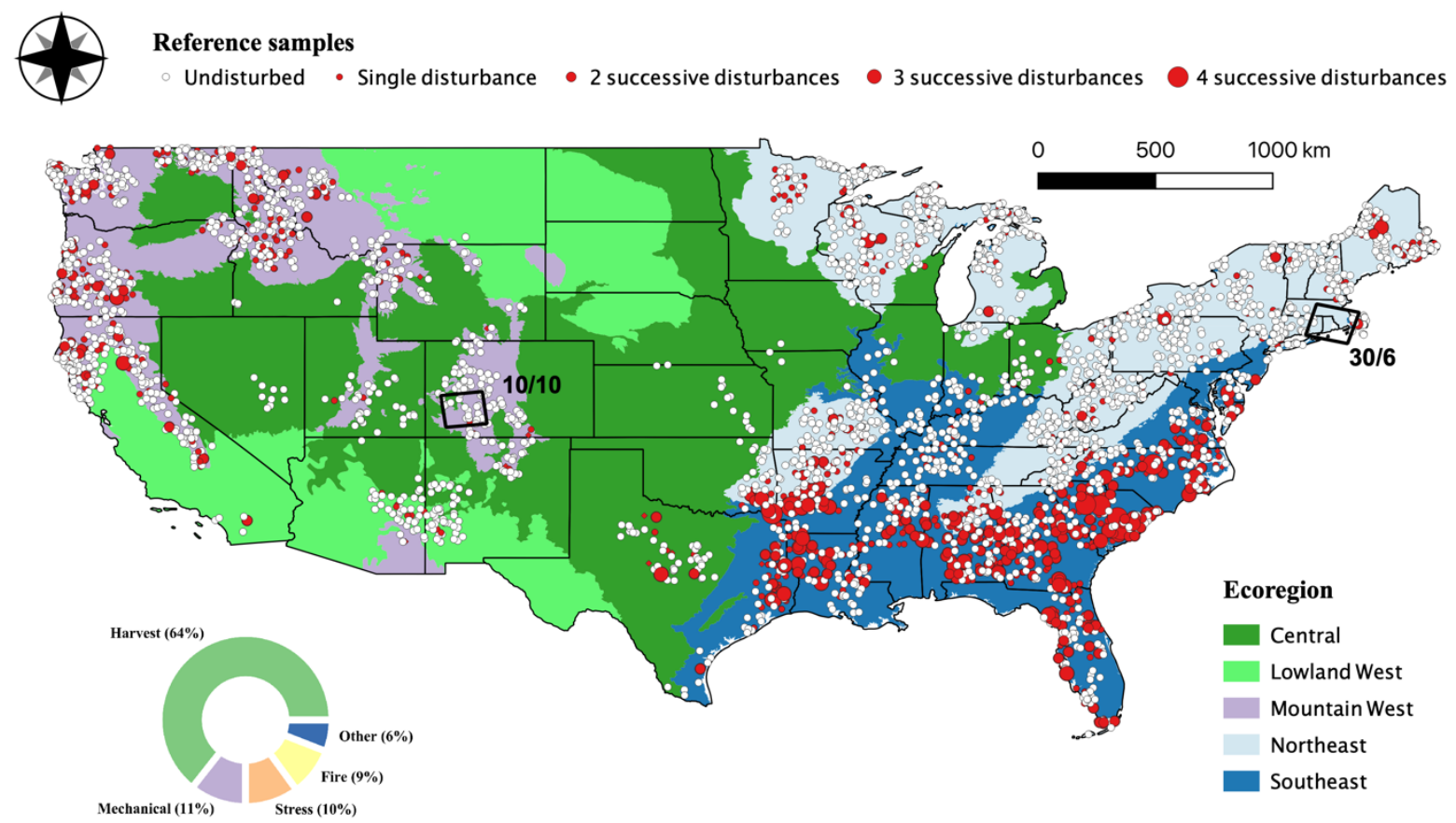

418 Fig. 6: the spatial distribution of 3,782 reference sites, and two Landsat ARD scenes chosen as the study areas. The reference sites include 2,704 undisturbed plots ('white circles') and 1,078 disturbed plots (red circles), which were collected using a stratified sampling based on the ecoregions across the United States. There are 1,413 disturbance occurrences in total, and the disturbance categories were dominated by the disturbance category 'Harvest' $(63.9 \%)$, followed by 'Mechanical' (10.5\%) and 'Stress' (9.9\%). Landsat ARD 10/10 is located in the south Colorado including a region where Papoose fire happened in 2013; Landsat ARD 30/6 is located in the New England, including a site affected by gypsy moth infestation in 2016 and 2017.

Considering that different algorithms may have different sensitivities to disturbance magnitudes based on

428 change probability thresholds, the omission and commission rates based on a series of change probability, 429 namely $0.90,0.925,0.95,0.975,0.99$, were chosen for accuracy assessment. The overall performance is 430 evaluated using the F1 score, because it provides a balanced assessment for omission and commission rates.

431 The definition of omission, commission rates and F1 score are the same as Zhu et al. (2019). 
This preprint has been submitted to Remote Sensing of Environment for peer review

$432 \quad F 1$ score $=\frac{(1-\text { commission }) *(1-\text { omission })}{2-\text { commission-omission }}$

433 In addition to quantitative accuracy assessment, qualitative map-based comparisons are performed by

434 evaluating break year maps detected by two algorithms. We chose two Landsat ARD tiles that were 435 respectively affected by fire and insect disturbance. The fire site sits at the San Juan and Rio Grande 436 National Forests in southwestern Colorado, where the second largest fire in Colorado history, West Fork 437 Fire Complex, burned 438 square kilometers of forested in June and July 2013 (Verdin et al., 2013). The 438 burned area was dominated by Engelmann spruce (Picea engelmannii) and Subalpine fir (Abies lasiocarpa) 439 (Carlson et al., 2017), and the burn severity was moderate or high for 59 percent of the area within the burn 440 perimeter (Verdin et al., 2013). The West Fork Complex consisted of three lightning-caused wildfires, 441 namely West Fork, Papoose and Windy Pass. Our study site is located Landsat ARD tile path/row 10/10 442 (see the black rectangle in Fig. 6), which covers all the burned region of Papoose fire (see Fig.9). Caused 443 by lightning, the Papoose fire starts on June 19, 2013, spread with a southeast direction, and was 444 considerably dampened by precipitation on July 19, 2013 (Cyphers et al., 2019; United States Department 445 of, 2014). All Landsat ARD images between 1996 and 2019 (25 years) were downloaded for analysis. A 446 fire perimeter map for this specific fire provided by GeoMAC mapping application 447 (https://rmgsc.cr.usgs.gov/outgoing/GeoMAC/) was used as our reference map. Directed by United States

448 Geological Survey (USGS), GeoMAC updates the fire perimeter data based upon inputs from incident 449 intelligence sources, GPS data, infrared imagery from satellite platform (Walters et al., 2011), and provides 450 the most accurate perimeter map for this fire to our best knowledge.

451 The second disturbance site was chosen for gypsy moth (Lymantria dispar) infestation in Southern New 452 England, which covers southern Massachusetts, northeast Connecticut, and northwest Rhode Island. This 453 is a peri-urban region with $56.7 \%$ forest cover (i.e., $34.8 \%$ hardwood, $17.8 \%$ mixed and $4.1 \%$ conifer), and $45426.7 \%$ developed area and 10.8\% wetlands (See Fig. 8D). This study area contains the locations that 455 experienced from gypsy moth outbreak that started in 2015, spiked in 2017 (Pasquarella et al., 2018). 456 Compared with fire or harvest disturbance, insect disturbances often lead to short-term or gradual spectral 
This preprint has been submitted to Remote Sensing of Environment for peer review

457 changes (Vogelmann et al., 2016). A gypsy moth infestation can consume a large quantity of foliage and

458 sometimes causes a near-total defoliation over a season or two (Townsend et al., 2004), but often starts to

459 recover very soon for the following year, so the change signal is commonly ephemeral (Vogelmann et al.,

460 2016). In addition, infested stands typically consist of two or more dominant tree species usually

461 represented by multi-aged and multi-sized populations(Hart and Veblen, 2015), making the detection all

462 the more challenging because of mixed spectral response from various tree species. All Landsat ARD

463 images for path/row 30/6 in recent 10 years (2010-2019) were downloaded and preprocessed. The Aerial

464 Detection Survey (ADS) data ${ }^{6}$ were used as reference dataset. The ADS data are polygon-based forest

465 health maps from visually-defined polygons for a variety of specific insects and disease, annually reported

466 by United States Department of Agriculture (USDA). It has been reported that the ADS data have high

467 omission and commission rates respectively as 32\% and 33\% (Johnson and Ross, 2008). The ADS data,

468 however, are a valuable source for indicating an approximate region for forest disease at a broad-scale

469 (minimum mapping unit as 5 ha) (Hart and Veblen, 2015; Preisler et al., 2012), and the timing of disease

470 occurrence.

\section{$471 \quad$ 5. Results}

\subsection{Quantitative accuracy assessment}

473 We tested COLD and S-CCD against a holdout validation set $(n=1891)$ for a series of threshold probability.

474 The omission, commission and F1 score (see annotation around markers) for each test have been shown in

475 Fig. 7A. S-CCD outperforms COLD at lower thresholds at 0.90, 0.925 and 0.95 for F1 score, while COLD

476 is slightly better at higher thresholds at 0.975 and 0.99 . The best accuracy they reached are close: S-CCD

477 achieve the best performance as a F1 score of 0.793 (0.95 threshold probability, $20.1 \%$ omission and $21.4 \%$

478 commission errors), while COLD reaches the best accuracy as F1 score of 0.789 (0.975 threshold

479 probability, $24.6 \%$ omission and $17.2 \%$ commission errors).

${ }^{6}$ http://www.foresthealth.fs.usda.gov/portal/Flex/IDS 
This preprint has been submitted to Remote Sensing of Environment for peer review

480 We also evaluated the performance of S-CCD and COLD against seven forest disturbance categories with 481 a threshold probability of 0.95 (Fig. 7B). Though COLD showed the best F1 score at the probability 0.975 , 482 a probability threshold of 0.95 is chosen considering: 1) to keep consistent for comparison; and 2) COLD 483 achieved a better balance of omission and commission errors at probability 0.95. As Fig. 7B shows, S-CCD 484 and COLD have very close omission errors for 'Harvest', 'Mechanical' and 'Wind'; S-CCD achieved lower 485 omission error rate in the disturbance category 'Stress' (16.4\% v.s. $24.6 \%)$ and 'Fire' (31.3\% v.s. 35.8\%), 486 but is worse in 'Hydrology' (12.5\% v.s. 37.5\%) and 'Other' (32.5\% v.s. $22.5 \%)$. Surprisingly, the two 487 approaches both have relatively high omission errors for 'fire' disturbance, which is often known for 488 causing large change magnitude from satellite images (Cohen et al., 2016). The reason is that some low489 severity/underground fire cases are included in the reference dataset, which causes only subtle spectral 490 change magnitude. The extremely low error rate for 'Wind' category is because the number of 'Wind' 491 samples $(n=13)$ is small and might be underrepresented in our database. Note that per-category accuracy 492 results of COLD for our tests are distinct with the results in Section 4.1 in (Zhu et al., 2019), which is 493 mainly due to 1 ) we used a lower threshold, 0.95 , instead of $0.99 ; 2$ ) different choices for training and 494 holdout reference samples due to random split; 3) only forest samples are analyzed. 

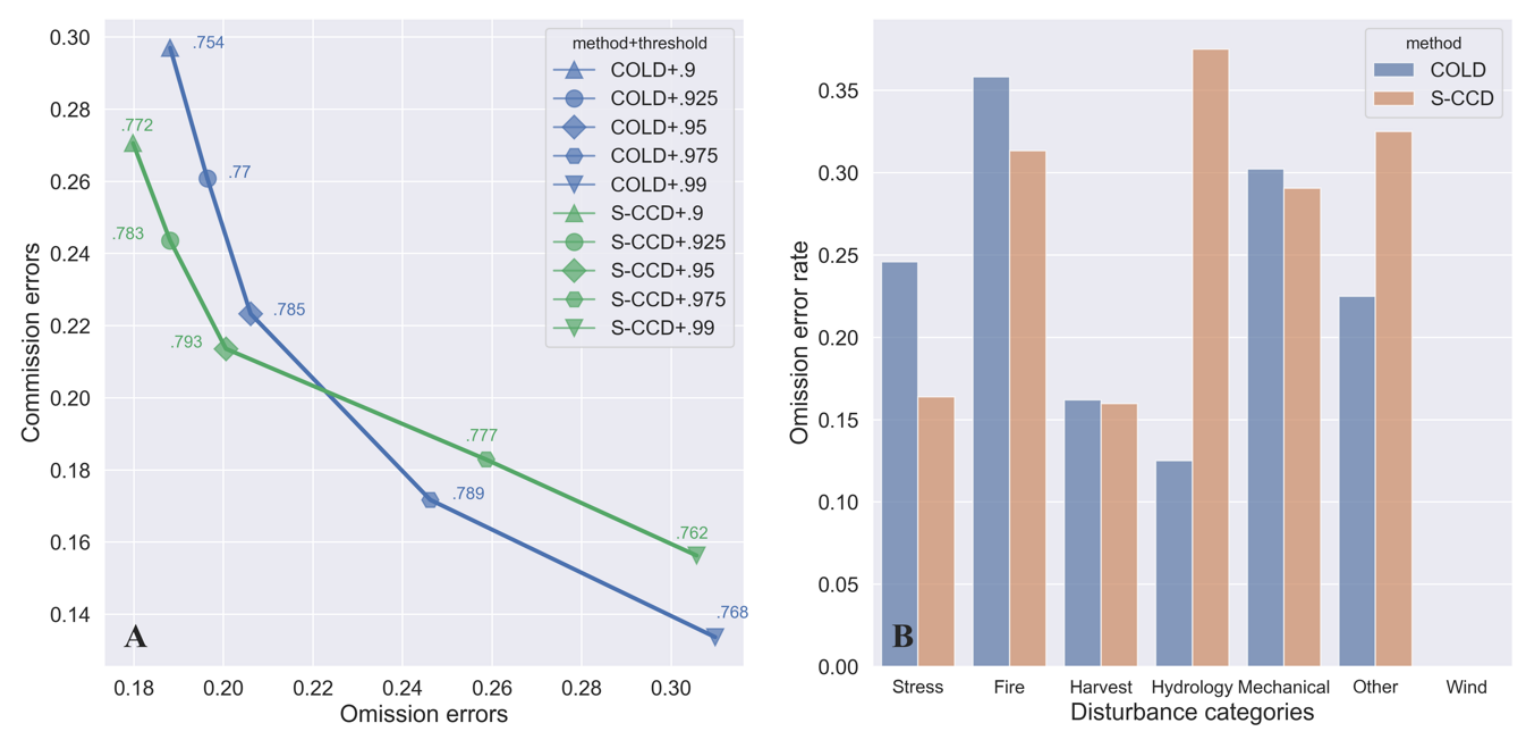

Fig. 7. Quantitative accuracy assessment results of COLD and S-CCD based on a holdout reference sample set $(n=1,891)$. A) shows omission, commission errors using a series of probability thresholds as $0.9,0.925,0.95,0.975$ and 0.99 . The $F 1$ score is used to evaluate the overall accuracy combining omission and commission errors, annotated around each marker. B) reports the omission errors of COLD and S-CCD for each disturbance category.

\subsection{Qualitative accuracy assessment}

504 For qualitative comparison, S-CCD and COLD were implemented to detect timing of forest disturbances respectively for Papoose fire and gypsy moth infestation. A consistent probability threshold as 0.95 was applied for both algorithms.

Fig. 8 shows the detection results of S-CCD and COLD for the Papoose fire site. Visually, COLD and SCCD show very similar detection results for abrupt disturbance categories such as moderate/high-severity fire. As this region has been heavily affected by spruce beetle since 2010 (Hart and Veblen, 2015), we selected the breakpoints only detected in 2013, that is the year for Papoose fire, with an assumption that

511 most of these breakpoints are associated with the fire, not spruce beetle attack. To assess accuracy of timings

512 for these breakpoints, we outputted month maps for the breakpoints detected in 2013. These breakpoints

513 mostly occurred in June and July (see Fig. 8A and B), which are well matched with the active temporal 
This preprint has been submitted to Remote Sensing of Environment for peer review

514 window of Papoose fire on the historical records (Cyphers et al., 2019; United States Department of, 2014),

515 indicating high temporal accuracy of detected breakpoints for both methods. To further evaluate spatial

516 accuracy for damage mapping, we compare two detection maps with GeoMAC fire perimeter map (Fig.

517 8C). GeoMAC contains some commission errors for mapping fire damage areas that are actually bare land

518 from High-resolution Satellite imagery, while COLD and S-CCD both accurately labeled them as 'no-attack'

519 (see the example of Fig. 8D, E and F). It is noteworthy that Landsat 7 related scan-line corrector (SLC)

520 artifacts can be both seen in the two breakpoint month maps, where the 'month of disturbance' was labeled

521 as July, one month later than it should be. The disturbance dates detected by COLD and S-CCD may show

522 several days to a month as delayed, if the pixels happen to have data gaps right after the disturbance dates

523 due to the SLC-off issue.

524

525 


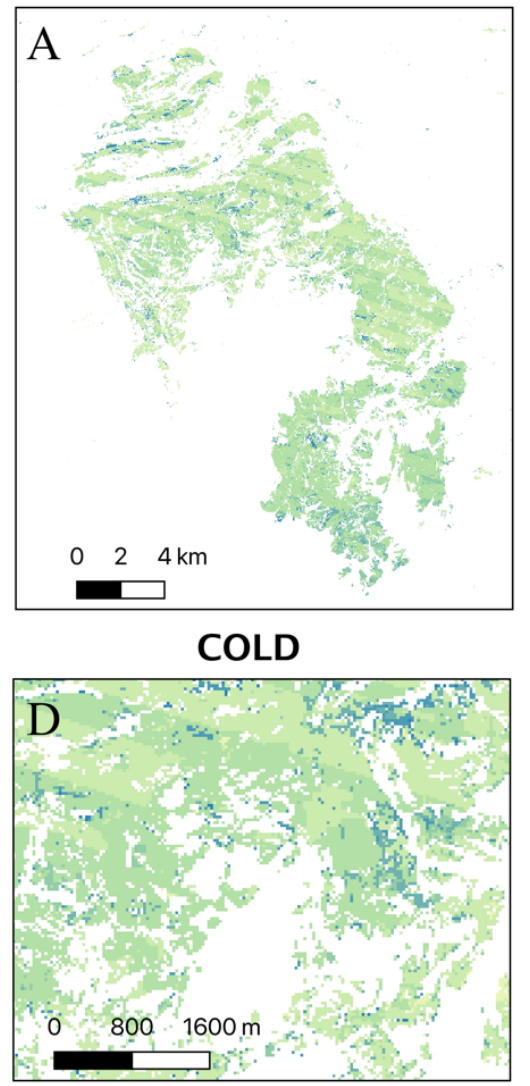

COLD

\section{Legend}

Month of breakpoints for $A$ and $B$

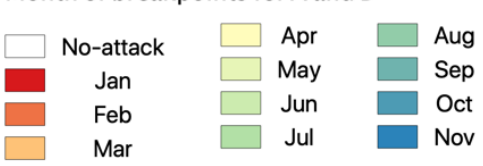

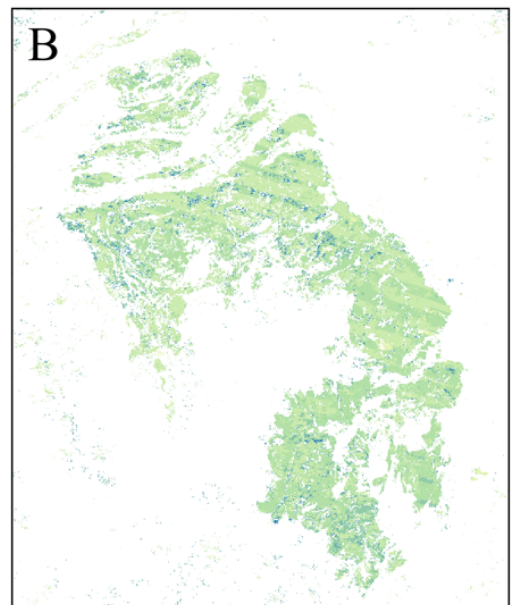
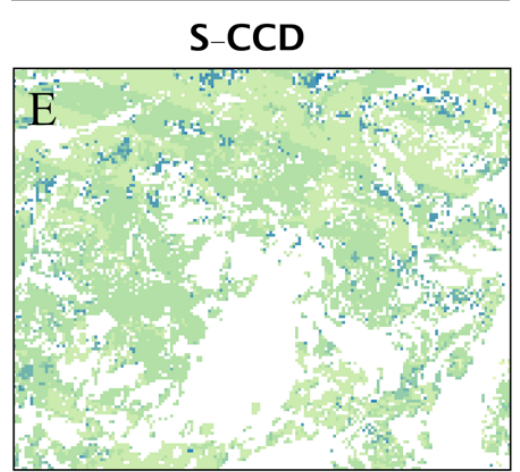

S-CCD
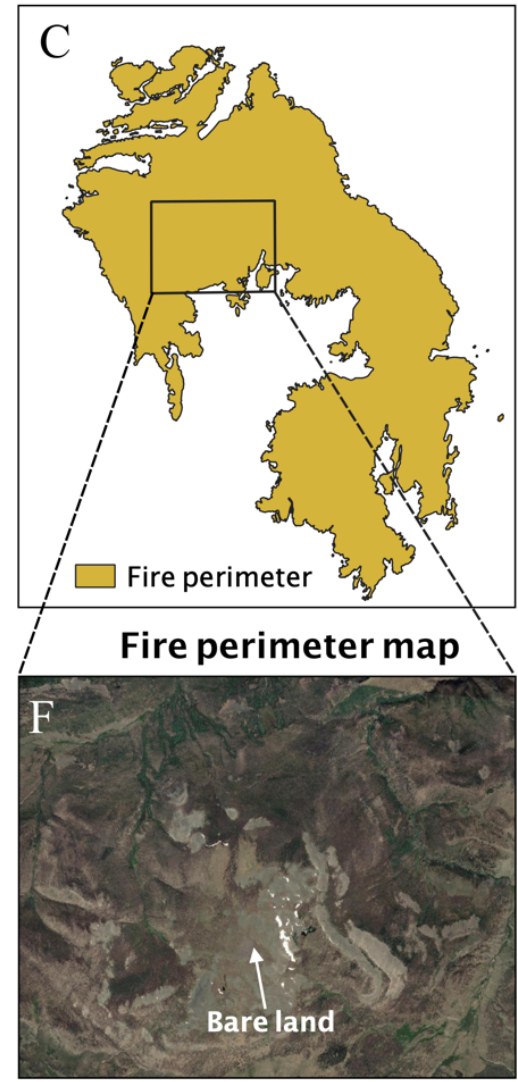

2016 Google image

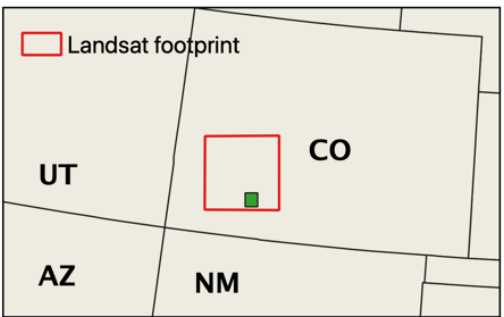

Fig. 8. Qualitative comparison for Papoose fire site (Landsat ARD 10/10) in Southeast Colorado among COLD (A), S-CCD (B) and GeoMAC fire perimeter map (C). COLD and S-CCD both use the probability threshold as $\mathbf{0 . 9 5}$. The color for $A$ ) and B) denotes the month of breakpoints detected in 2013: the spectrum ranging from red to blue corresponds to months of breakpoints from January to November; the white are no-attack regions in 2013. D), E) and F) shows an example region that COLD and S-CCD both accurately delineated non-attack bare land while GeoMAC over-detects it as fire region.

For the gypsy moth scene (Fig. 9), we compared years of breakpoints detected with Aerial Surveying 
This preprint has been submitted to Remote Sensing of Environment for peer review

537 insect-induced tree mortality, the reference map chose the disturbance year as the first year that a

538 disturbance captured by the ADS. Most disturbance events detected by COLD and S-CCD are concentrated

539 at the deciduous forest cover regions of the 2016 NLCD map (Fig. 9D). The disturbance years indicated by

540 two algorithms matched that of the ADS data, which are primarily the year of 2016 and 2017. This finding

541 is consistent with the historical record that a major outbreak began in early summer 2016, led by a series of

542 unusually dry springs (2014-2016) (Pasquarella et al., 2017). Admittedly, both algorithms yield much

543 fewer affected regions than that the ADS shows. Considering that Pasquarella et al. (2017) got the same

544 result for their study in the Southern New England region, those omission errors might be due to

545 overestimation of tree mortality regions in aerial sketch mapping. For comparative analysis, we found that

546 COLD missed some regions affected by gypsy moth (e.g., the dashed blue circle in Fig. 9A), while S-CCD

547 found most parts of the same region as forest disturbance. The general locations and the disturbance years

548 for extra regions detected by S-CCD have agreement with the ADS data (see the dashed circle in Fig.9C),

549 proving that these extra breakpoints detected by S-CCD for this region are related to forest damage caused

550 by gypsy moth. In addition, the damaged trees, which appears to be 'gray' color, can be clearly identified

551 from 2018 high-resolution Google satellite image (see Fig. 9G), and the geometric shape of damaged area

552 in the high-resolution imagery align with that of the disturbance region detected by S-CCD (Fig. 9F). 
Legend
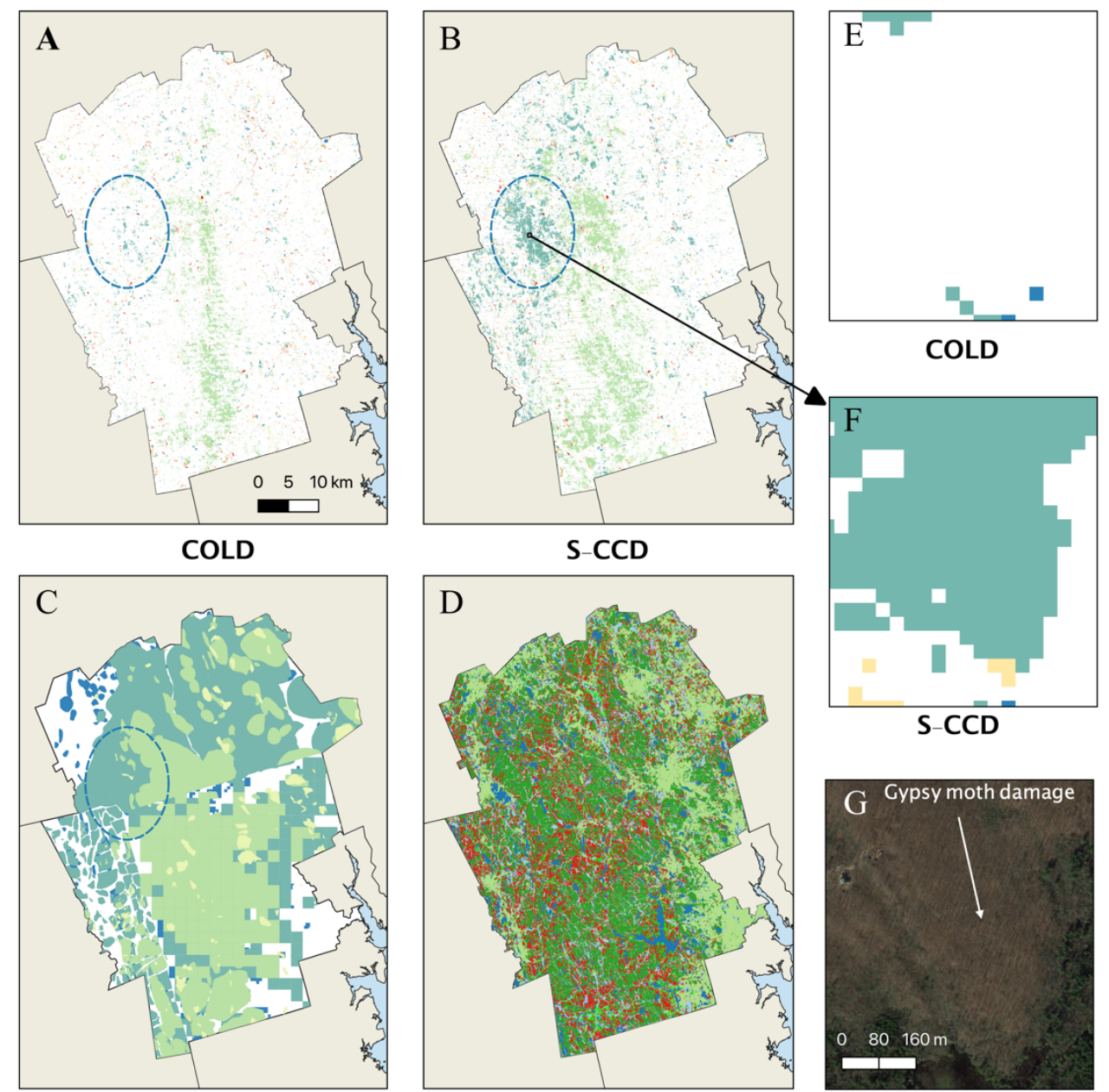

ADS map

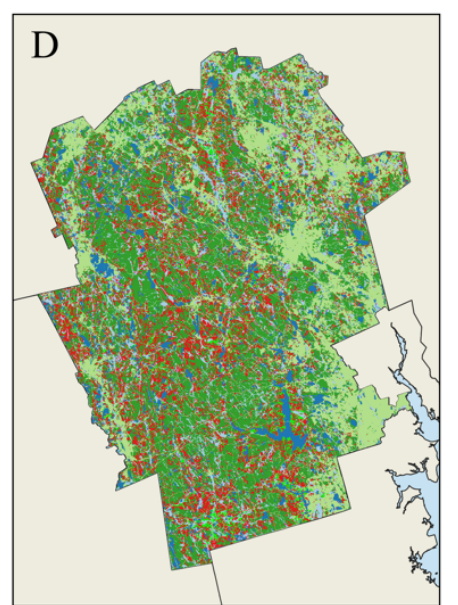

NLCD Land cover map

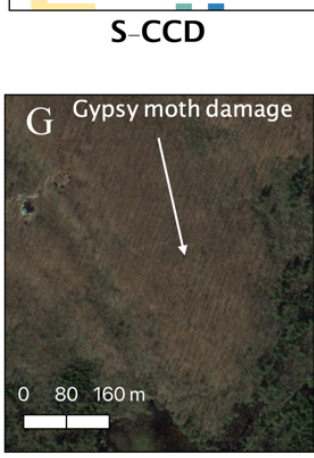

2018 Google image

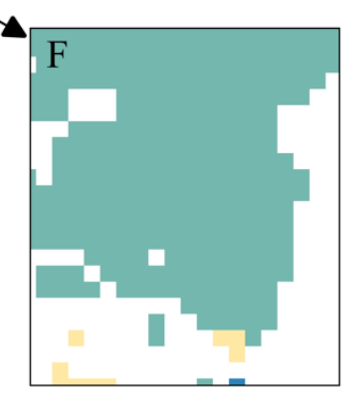

Break years for $A, B$ and $C$

$\square$ No_attack

2011

2012

$\square 2013$

$\square 2014$

$\square 2015$

$\square 2016$

$\square 2017$

2018

NLCD land types for D

$\square$ Developed

Deciduous

$\square$ Evergreen

Mixed

$\square$ Wetland

Others
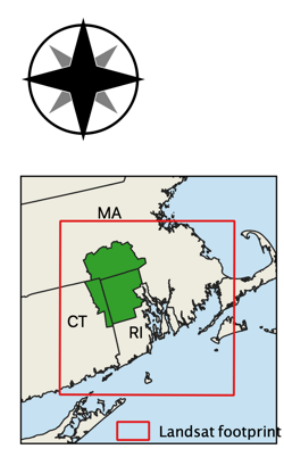

Fig. 9. Qualitative comparison for gypsy moth site (Landsat ARD 30/6) in Southern New England among COLD (A), S-CCD (B) and the ADS reference map (C). (D) shows the land cover types from NLCD 2016. COLD and S-CCD both use the probability threshold as 0.95 . The color for (A), (B) and $(C)$ denote the disturbance year indicated by each data source: the spectrum ranging from red to blue corresponds to the years from 2011 to 2018. Fig.9 E), F) and G) shows an example region that COLD missed gypsy moth damage which can be clearly identified in the high-resolution Google imagery.

\subsection{Efficiency test and software implementation}

We implemented COLD and S-CCD in the high-performance C programming language. The C package named 'S-CCD' is downloadable from https://github.com/SuYe99/s-ccd (the repo was temporally set to be 
This preprint has been submitted to Remote Sensing of Environment for peer review

566 interface is also provided by the package. The software implemented a shared memory parallelization for 567 COLD and S-CCD under a Linux/MacOS desktop environment, and can be easily adapted for a High568 Performance Computing (HPC) environment. It is noteworthy that our C-based COLD has been 15-20 569 times faster than the original MATLAB-based implementation.

570 To test the efficiency for C-based COLD and S-CCD, we used a 'dummy' Landsat ARD scanline which is 571 a standard sample set of 5,000 pixel-based time series plots selected from our reference sample set. The 572 sample set consists of 3,782 forest plots and 1,218 non-forest plots. To uncover the effects of monitoring 573 span on the speed, we pruned each time series into three different lengths of time series records, that is 10 574 years (2008-2017), 20 years (1998-2017) and 34 years (1984-2017). The results are summarized in Fig. 575 10. S-CCD can achieve up to 4.4 times faster than the C-based COLD (with 34-year time series records). 576 With the length of records decreasing, the efficiency improvement of S-CCD over COLD declines, as $\sim 3.5$ 577 times at 20-year time series records, and $\sim 1.8$ time faster at 10-year time series records. This is because S578 CCD improves efficiency mainly at the step of the model update; a longer time series needs a greater 579 number for model updates to complete a detection, and hence more significant efficiency improvement 580 made by S-CCD. For a standard 20-year Landsat ARD time series, our S-CCD program takes only $\sim 6$ 581 minutes to finish a detection for a 5000-pixel scanline, and 500 computing hours for a Landsat ARD scene. 


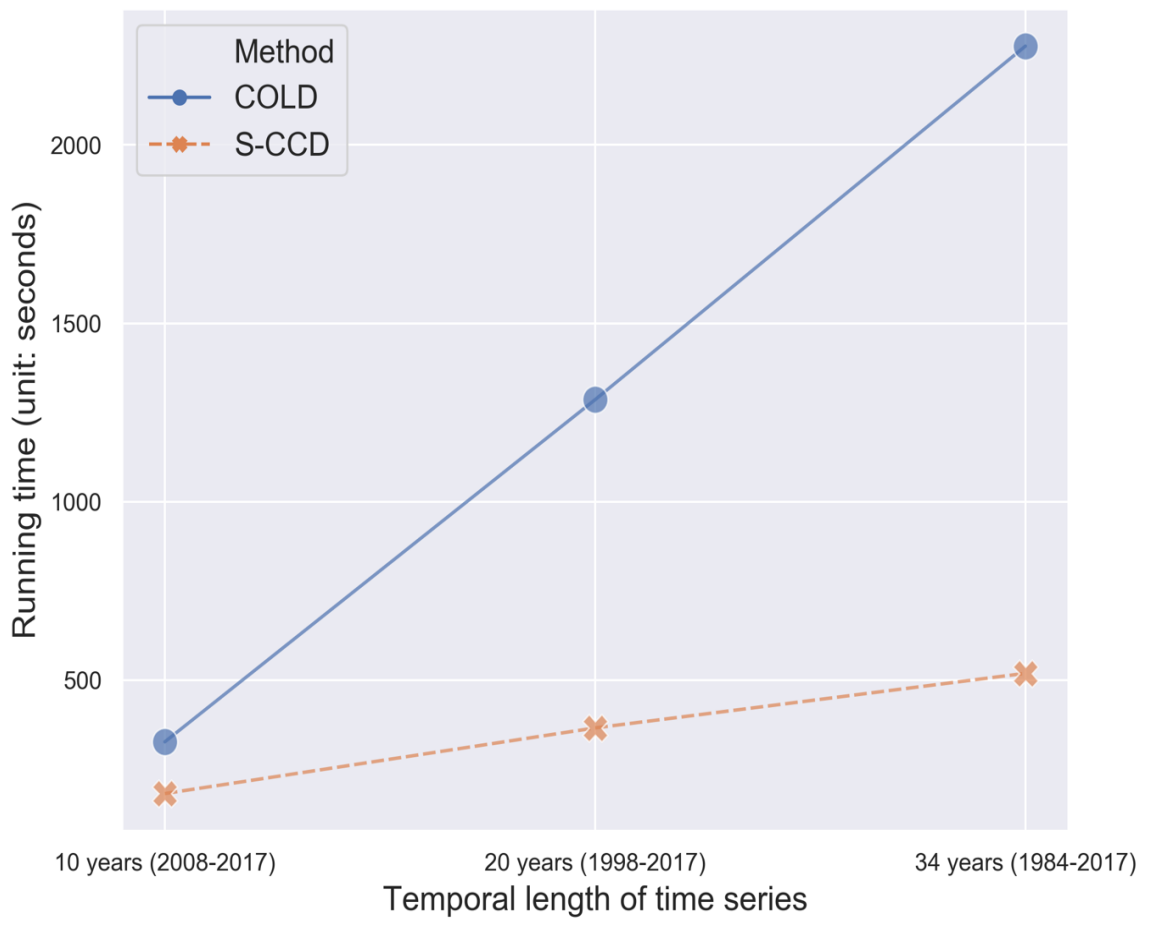

Fig. 10. The result for the efficiency test based on a dummy Landsat ARD scanline (5,000 sample pixels). Each time series are pruned as three versions for time series records, that is 10 years (20082017), 20 years (1998-2017), 34 years (1984-2017). The result shows that the efficiency of S-CCD was increased as the length of time series increased, and can be up to 4.4 times faster than COLD. The CPU is Intel(R) Core (TM) i7-4790, 3.60GHz.

590 The quantitative accuracy assessment indicates that the differences between the best accuracy achieved by 591 S-CCD and COLD are only $0.4 \%$ (see Fig. 7A, 0.793 v.s. 0.789). The primary reason is that our plot 592 database is generated from random sampling over a nation-wide region, for which $63 \%$ of the forest 593 disturbances are harvesting activities; the two approaches have very similar performance for detecting 594 strong spectral signals yielded by those disturbances with medium- or high-severity and homogeneous tree 595 damages such as harvest disturbances (omission errors: 16\% v.s. 16.2\%). Our map-based evaluation for 596 Papoose fire case can confirm this conclusion. 
This preprint has been submitted to Remote Sensing of Environment for peer review

597 We noticed that, however, S-CCD achieved noticeably less omission errors than COLD for those lower-

598 magnitude disturbances such as drought stress and low-severity fire (see Fig. 7b). This finding was

599 confirmed by our qualitative comparison for the case study of gypsy moths. The reason is three-fold. First,

600 S-CCD allows for known changes in the structure of the system over time, and often can achieve a better

601 model fitting than COLD that assumes the rigidity of "linear trend + harmonic cycles". We calculated the

602 average RMSE for each band using all our 3,782 samples. The results, as shown in Table 1, indicate that

603 S-CCD has generally lower average RMSE than COLD for all seven spectral bands other than NIR and

604 thermal bands (thermal band is not used to compute change magnitudes for both approaches, and thereby

605 has no effects on break detection). As the two approaches both evaluated change magnitude relative to the

606 RMSE, the lower RMSE means that S-CCD model is more sensitive to those low-magnitude spectral

607 changes.

Table 1: the average RMSE of COLD and S-CCD for each band using 3,782 forest samples across the conterminous United States (the bold columns are the two bands that S-CCD has higher RMSE than COLD)

\begin{tabular}{llllllll}
\hline Band & Blue & Green & Red & NIR & SWIR1 & SWIR2 & Thermal \\
\hline COLD & 122.682 & 117.127 & 122.473 & $\mathbf{2 7 3 . 3 3 6}$ & 197.937 & 151.032 & $\mathbf{3 8 9 . 2 2 7}$ \\
S-CCD & 119.061 & 110.761 & 118.476 & $\mathbf{2 7 9 . 4 7 8}$ & 191.518 & 150.098 & $\mathbf{4 0 1 . 0 6 1}$ \\
\hline
\end{tabular}

612 Second, S-CCD uses a new temporally-adjusted peek window, for which the window width is defined as

613 calendar days, not a fixed observation number. Fig.11 is an example of NIR-based time series from our

614 gypsy moth case: S-CCD successfully detected the gypsy moth attack with a breakpoint as '2016-05-17'

615 (the black solid line in Fig. 11B) while missed by the COLD. COLD employs a fixed number of 12

616 observations for its peak window as the median revisit days is 8 days for this case (see Formula S2 in the

617 supplementary material of (Zhu et al., 2019)). If the peek window spans over a period of sparse clear

618 observation such as May and June on 2016, the peek window has to be extended to later months in order to

619 include enough clear observations. Differently, S-CCD defines the peek window as the minimum width of 
This preprint has been submitted to Remote Sensing of Environment for peer review

80 calendar days, so when the monitoring stands at '2016-05-17' (the breakpoint detected), the peek window of S-CCD stops at the date '2016-07-20' (see the red dashed line in Fig. 11B), roughly one month earlier than that of COLD ('2016-08-21', see the red dashed line in Fig. 11A). A gradual decrease in NIR band ('greendown') is commonly observed during the later summer in deciduous broadleaf forests (Elmore et al., 2012; Melaas et al., 2013), which is also shown in the harmonic modeling curve of Fig 11A. As a result, the damage signal was overridden by normal phenological changes at the end of the peek window for COLD, causing the breakpoint to be missed for detection.
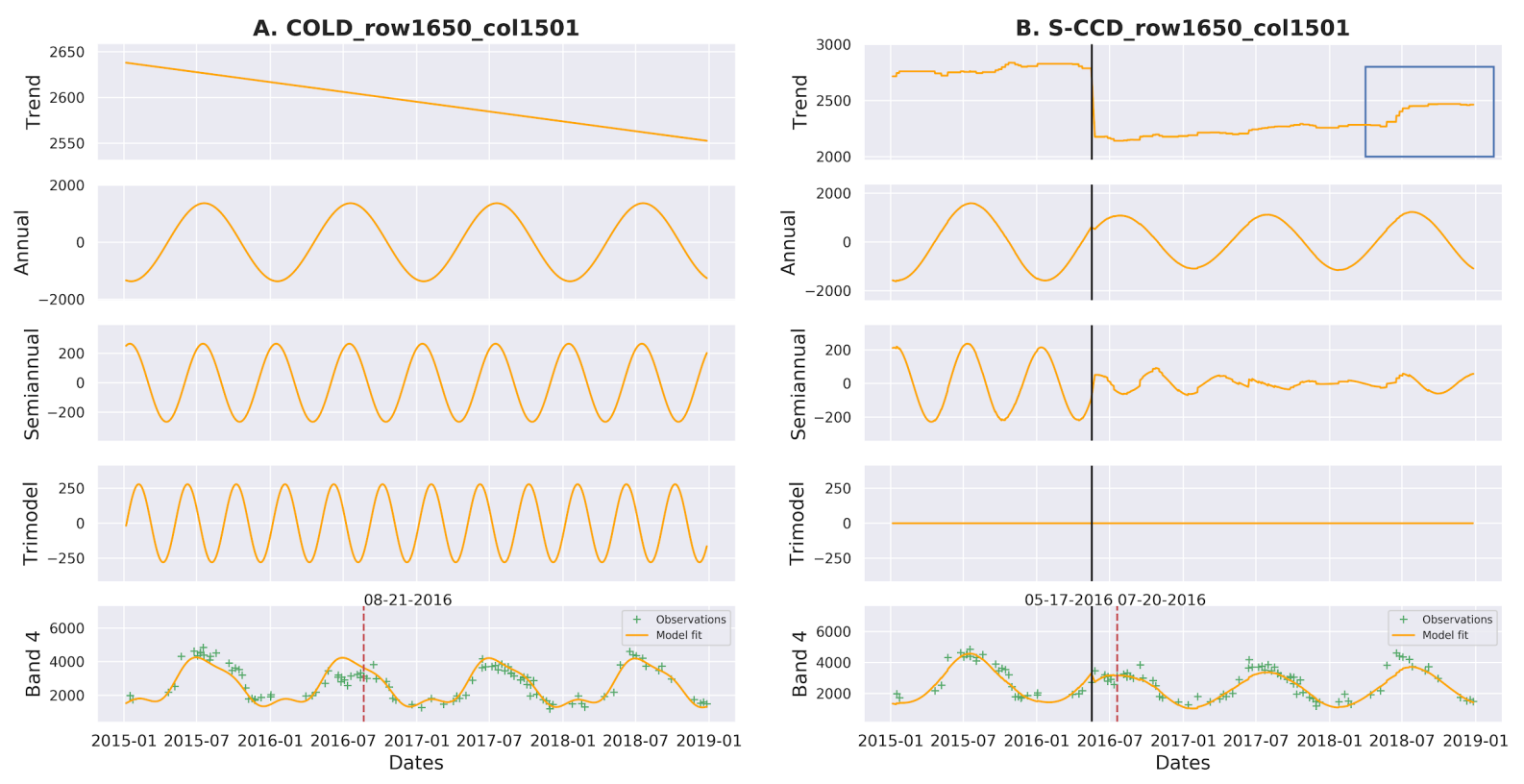

Fig. 11. An example where S-CCD detects the breakpoint that was missed by COLD, from a gypsy moth attack site. The black line: the breakpoint detected. The red dashed lines: the ending dates of the peek window for each method, when the monitoring stands at '05-17-2016' (the peak window of S-CCD is finished with one month earlier than COLD). The blue rectangle shows the recent signal for increasing NIR associated with forest regeneration, which is revealed by 'trend' component of S-CCD ( The trimodel component is set as a constant ' 0 ' for S-CCD here because the trimodel component has been excluded for S-CCD).

Third, if COLD/CCDC misses observations at the beginning of a gradual change, the algorithm will

637 incorporate these 'change' observations to refit the model with a slight slope associated with 'degradation'.

638 This slope results in lower change magnitudes for subsequent observations, possibly causing a complete 
This preprint has been submitted to Remote Sensing of Environment for peer review

639 missing detection for the break (Bullock et al., 2019). Owing to no slope component in our predefined 640 structure (see Equation 6), S-CCD, however, assumes that the trend state to be constant for future, rather 641 than continue degrading, thereby owns a chance to detect the breakpoint later.

642 S-CCD also provides a rich set of information for help elucidate short-term fluctuations via its continuous 643 varying expression for trend and seasonality. For the example of Fig.11, the NIR trend component for S644 CCD appears to be increasing after the year 2018 (see the blue rectangle in Fig.11B), indicating the 645 greenness is recovering. COLD fails to unveil these subtle signals because it generalizes linear trend and 646 seasonality using a simple harmonic model. S-CCD is more informative for probing such-like short-term 647 and subtle signals which might have important ecological implications, owing to its ability to model more 648 complex nonlinear temporal dynamics. It is worth mentioning that our software package provides this 649 functionality of visualization for plotting dynamics of each state.

650 Another obvious advantage of S-CCD is that it is a completely online monitoring algorithm, which can be 651 directly used for near real-time forest monitoring. S-CCD improves several steps of COLD and enables a 652 continuous monitoring in a completely recursive form, such as the adjusted peek window, recursive 653 temporal RMSE. More importantly, the core technique for S-CCD, the Kalman filter, is a powerful real654 time algorithm well known for its high computational efficiency and short-memory requirement. For COLD, 655 limited by its manner for re-constructing the model per observation, it requires reading all images into the 656 system to rebuild harmonic curves for each new observation. Instead, S-CCD just needs to update those 657 parameter files (e.g., states and covariance) once the initialization is finished, which can reduce $\sim 95 \%$ of 658 data inputs for monitoring each new observation. In addition, the Kalman filter has an extremely simple 659 mathematical treatment for dealing with missing data, therefore is an ideal tool for processing remote 660 sensing time series which often have irregular temporal intervals between successive observations.

661 A possible concern is that S-CCD assumes a consistent structure of time series for trend, annual and semi662 annual harmonic items (equal to 6-coefficient harmonic models), while Zhu et al. (2019) suggested using a 663 harmonic model with a maximum coefficient as 8, including trend, annual, semi-annual and trimodal 
This preprint has been submitted to Remote Sensing of Environment for peer review

664 harmonic items (frequency $=1 / 3$ year). As such, we tested adding an additional trimodal cycle component 665 into the presumed time series structure of S-CCD, and found that the best F1 score decreased from 0.795 666 to 0.706 for S-CCD (see Fig. S4 in the supplementary material). In a harmonic regression, trimodal 667 component can be viewed as an additional modifier to annual and semi-annual curves (Eastman et al., 2009).

668 The state space model adopted a totally different strategy to resolve unexplained variance from annual and 669 semi-annual curves: the state space model assumes that the trend and seasonality states are evolving as time 670 goes by (see Fig. 2B), instead of following a fixed set of harmonic coefficients, thus the unexplained 671 variances can be 'ingested' immediately by changes in structure over time. Another reason might be that 672 we focus on forest disturbance detection for this study, while the trimodal component is often found to be 673 more useful for modeling cropland dynamics.

674 Admittedly, COLD is designed for detecting/characterizing all types of land disturbances, not limited to 675 forest disturbances. We tested S-CCD against a reference dataset for comprehensive land types, and got a 676 slightly lower F1 score compared with COLD (0.69 vs 0.71). Our test shows that S-CCD performs less 677 ideal under a highly-fluctuated environment, and is more prone to over-detection due to those ephemeral 678 changes that have a high change magnitude, such as moisture change for grassland/bare land and 679 agricultural rotation. To alleviate this issue, our software package enables users to specify a mask for 680 focused study area, which can exclude the regions where uninterested land change occur and greatly 681 improve processing efficiency as well. Yet, there is still much studies that is needed to analyze 682 characteristics of detected breaks and select breaks only linked to targeted physical processes. Another 683 reason for the unsatisfactory results is that we often have multiple historical data sources to confirm forest 684 disturbances such as the ADS and the LANDFIRE products, but reliable references for the other non-forest 685 land disturbance are lacking. The quality of our non-forest land samples might affect the final accuracy 686 result. Our future work will be directed into 1) modifying S-CCD to accommodate other applications such 687 as agricultural shifts and urban expansion, and 2) tuning the algorithm for the optimal parameters, such as 
This preprint has been submitted to Remote Sensing of Environment for peer review

688

689

690

691 We presented an improved time series framework, Stochastic Continuous Change Detection (S-CCD), for

692 near real-time forest disturbance monitoring. The new approach introduces the state space model into the

693 current framework for Continuous Monitoring of Land Disturbance (COLD), to facilitate a complete near

694 real-time analytics of forest dynamics and improve computational efficiency. S-CCD provides an accurate

695 mapping for timing and change magnitude of forest disturbance, and uncovers complex nonlinear dynamics

696 from time series data. Especially S-CCD can improve the monitoring for those disturbances that induce

697 subtle spectral changes.

698

699

700

701

702

703

704

705

706

707

708

709

\section{Acknowledgement}

This work has been supported with funding from American Society of Photogrammetry and Remote Sensing (ASPRS) William A. Fisher Scholarship and Albert, and Norma and Howard Geller '77 Endowed Research Awards to Su Ye. We also gratefully acknowledge the support of USGS-NASA Landsat Science Team (LST) Program for Toward Near Real-time Monitoring and Characterization of Land Surface Change for the Conterminous US (140G0119C0008) to Zhe Zhu. 
This preprint has been submitted to Remote Sensing of Environment for peer review

710

711

712 The system matrices for 'trend+annual+semi-annual' time series structural model are defined by

713

714

$Z=(1,1,0,1,0)$

$Q=\operatorname{diag}\left(\sigma_{\xi}^{2}, \sigma_{\omega, \text { annual }}^{2}, \sigma_{\omega^{*}, \text { annual }}^{2}, \sigma_{\omega, \text { semi }}^{2}, \sigma_{\omega^{*}, \text { semi }}^{2}\right)$

$715 T=\left[\begin{array}{ccccc}1 & 0 & 0 & 0 & 0 \\ 0 & \cos \left(\frac{2 \pi}{365.25}\right) & \sin \left(\frac{2 \pi}{365.25}\right) & 0 & 0 \\ 0 & -\sin \left(\frac{2 \pi}{365.25}\right) & \cos \left(\frac{2 \pi}{365.25}\right) & 0 & 0 \\ 0 & 0 & 0 & \cos \left(\frac{2 \pi}{2 * 365.25}\right) & \sin \left(\frac{2 \pi}{2 * 365.25}\right) \\ 0 & 0 & 0 & -\sin \left(\frac{2 \pi}{2 * 365.25}\right) & \cos \left(\frac{2 \pi}{2 * 365.25}\right)\end{array}\right]$
716

717 The Kalman filter recursion for the general Gaussian model of form are

$718 v_{t, i}=y_{t, i}-Z a_{t, i}$

$719 \quad F_{t, i}=Z P_{t, i} Z^{T}+H_{i}$

$720 \quad K_{t, i}=P_{t, i} Z^{T}$

$721 \quad a_{t \mid t, i}=a_{t, i}+K_{t, i} F_{t, i}^{-1} v_{t, i}$

$722 \quad a_{t+1, i}=T a_{t \mid t, i}$

723

$P_{t+1, i}=T\left(P_{t, i}-K_{t, i} K_{t, i}^{T} F_{t, i}^{-1}\right) T^{T}+Q_{i}$

724 Where

$725 y_{t, i}:$ the observation at time $t$ for band $i$

$726 v_{t, i}$ : the innovation, namely the difference between predicted and actual observations, at time $t$ for band $i$

$727 Z$ : the system matrix that determines which items in the state vector are included for the observation

$728 K_{t, i}$ : the Kalman gain which is the relative ratio of being assigned to the model update is from the

729 innovation at time $t$ for band $i$

$730 \quad P_{t, i}$ : the covariance matrix at time $t$ for band $i$

$731 a_{t \mid t, i}$ : the filtered states at time $t$ for band $i$

$732 F_{t, i}$ : the variance of the innovation $v_{t, i}$

$733 Q_{i}$ : the process noise for band $i$

$734 \quad H_{i}$ : the observational noise for band $i$

735

736 For missing observation, there is no innovation $v_{t, i}$. Therefore, the mathematical treatment for state and 737 covariance matrix updates can be simply put as

$738 a_{t+1, i}=T a_{t \mid t, i}$

$739 P_{t+1, i}=T P_{t, i} T^{T}+Q_{i}$ 
This preprint has been submitted to Remote Sensing of Environment for peer review

\section{Reference}

741 Allen, C.D., Breshears, D.D., \& McDowell, N.G. (2015). On underestimation of global vulnerability to

Brockwell, P.J., \& Davis, R.A. (2013). Introduction to Time Series and Forecasting. Springer Science \& Business Media

Brown, J.F., Tollerud, H.J., Barber, C.P., Zhou, Q., Dwyer, J.L., Vogelmann, J.E., Loveland, T.R., Woodcock, C.E., Stehman, S.V., Zhu, Z., Pengra, B.W., Smith, K., Horton, J.A., Xian, G., Auch, R.F., Sohl, T.L., Sayler, K.L., Gallant, A.L., Zelenak, D., Reker, R.R., \& Rover, J. (2019). Lessons learned implementing an operational continuous United States national land change monitoring capability: The Land Change Monitoring, Assessment, and Projection (LCMAP) approach. Remote Sensing of Environment, 111356

Bullock, E.L., Woodcock, C.E., \& Holden, C.E. (2019). Improved change monitoring using an ensemble of time series algorithms. Remote Sensing of Environment, 111165

Burkett, V.R., Wilcox, D.A., Stottlemyer, R., Barrow, W., Fagre, D., Baron, J., Price, J., Nielsen, J.L., Allen, C.D., Peterson, D.L., Ruggerone, G., \& Doyle, T. (2005). Nonlinear dynamics in ecosystem response to climatic change: Case studies and policy implications. Ecological Complexity, 2, 357-394

Carlson, A.R., Sibold, J.S., Assal, T.J., \& Negrón, J.F. (2017). Evidence of compounded disturbance effects on vegetation recovery following high-severity wildfire and spruce beetle outbreak. PLoS ONE, 12, e0181778

Claverie, M., Vermote, E.F., Franch, B., \& Masek, J.G. (2015). Evaluation of the Landsat-5 TM and Landsat-7 ETM+ surface reflectance products. Remote Sensing of Environment, 169, 390-403

Cohen, W.B., Healey, S.P., Yang, Z., Stehman, S.V., Brewer, C.K., Brooks, E.B., Gorelick, N., Huang, C., Hughes, M.J., \& Kennedy, R.E. (2017). How Similar Are Forest Disturbance Maps Derived from Different Landsat Time Series Algorithms? Forests, 8, 98

Cohen, W.B., Yang, Z., Stehman, S.V., Schroeder, T.A., Bell, D.M., Masek, J.G., Huang, C., \& Meigs, G.W. (2016). Forest disturbance across the conterminous United States from 1985-2012: The emerging dominance of forest decline. Forest ecology and management, 360, 242-252

Curran, L.M., \& Trigg, S.N. (2006). Sustainability science from space: quantifying forest disturbance and land-use dynamics in the Amazon. Proceedings of the National Academy of Sciences of the United States of America, 103, 12663-12664

Cyphers, L., Mackes, K., \& Duda, K. (2019). Timber Losses from West Fork Complex Fire in Southwest Colorado. For. Prod. J.

Dale, V.H., Joyce, L.A., McNulty, S., Neilson, R.P., Ayres, M.P., Flannigan, M.D., Hanson, P.J., Irland, L.C., Lugo, A.E., \& Peterson, C.J. (2001). Climate change and forest disturbances. BioScience, $51,723-734$

Davis, M.H.A., \& Vinter, R.B. (1985). Stochastic models. Stochastic Modelling and Control, 60-99

Durbin, J., \& Koopman, S.J. (2012). Time Series Analysis by State Space Methods: Second Edition. OUP Oxford

Dwyer, J., Roy, D., Sauer, B., Jenkerson, C., Zhang, H., \& Lymburner, L. (2018). Analysis Ready Data: Enabling Analysis of the Landsat Archive. Remote Sensing, 10 
This preprint has been submitted to Remote Sensing of Environment for peer review

781

782

783

784

785

786

787

788

789

790

791

792

793

794

795

796

797

798

799

800

801

802

803

804

805

806

807

808

809

810

811

812

813

814

815

816

817

818

819

820

821

Eastman, J.R., Sangermano, F., Ghimire, B., Zhu, H., Chen, H., Neeti, N., Cai, Y., Machado, E.A., \& Crema, S.C. (2009). Seasonal trend analysis of image time series. International journal of remote sensing, 30, 2721-2726

Eastman, J.R., Sangermano, F., Machado, E.A., Rogan, J., \& Anyamba, A. (2013). Global trends in seasonality of normalized difference vegetation index (NDVI), 1982-2011. Remote Sensing, 5, 4799-4818

Elmore, A.J., Guinn, S.M., Minsley, B.J., \& Richardson, A.D. (2012). Landscape controls on the timing of spring, autumn, and growing season length in mid-Atlantic forests. Global change biology, 18, 656-674

Hart, S.J., \& Veblen, T.T. (2015). Detection of spruce beetle-induced tree mortality using high-and medium-resolution remotely sensed imagery. Remote Sensing of Environment, 168, 134-145

Helske, J. (2016). KFAS: Exponential Family State Space Models in R. arXiv [stat.CO]

Jamali, S., Jönsson, P., Eklundh, L., Ardö, J., \& Seaquist, J. (2015). Detecting changes in vegetation trends using time series segmentation. Remote Sens. Environ., 156, 182-195

Johnson, E.W., \& Ross, J. (2008). Quantifying error in aerial survey data. Australian Forestry, 71, 216222

Ju, J., Roy, D.P., Vermote, E., Masek, J., \& Kovalskyy, V. (2012). Continental-scale validation of MODIS-based and LEDAPS Landsat ETM+ atmospheric correction methods. Remote Sensing of Environment, 122, 175-184

Kalman, R.E. (1960). A new approach to linear filtering and prediction problems. Int. J. Eng. Trans. A, $82,35-45$

Kautz, M., Meddens, A.J., Hall, R.J., \& Arneth, A. (2017). Biotic disturbances in Northern Hemisphere forests-a synthesis of recent data, uncertainties and implications for forest monitoring and modelling. Global Ecology and Biogeography, 26, 533-552

Kennedy, R.E., Andréfouët, S., Cohen, W.B., Gómez, C., Griffiths, P., Hais, M., Healey, S.P., Helmer, E.H., Hostert, P., Lyons, M.B., \& Others (2014). Bringing an ecological view of change to Landsat-based remote sensing. Front. Ecol. Environ., 12, 339-346

Kennedy, R.E., Yang, Z., \& Cohen, W.B. (2010). Detecting trends in forest disturbance and recovery using yearly Landsat time series: 1. LandTrendr-Temporal segmentation algorithms. Remote Sensing of Environment, 114, 2897-2910

Masek, J.G., Goward, S.N., Kennedy, R.E., Cohen, W.B., Moisen, G.G., Schleeweis, K., \& Huang, C. (2013). United States Forest Disturbance Trends Observed Using Landsat Time Series. Ecosystems, 16, 1087-1104

Masek, J.G., Huang, C., Wolfe, R., Cohen, W., Hall, F., Kutler, J., \& Nelson, P. (2008). North American forest disturbance mapped from a decadal Landsat record. Remote Sensing of Environment, 112, 2914-2926

Melaas, E.K., Friedl, M.A., \& Zhu, Z. (2013). Detecting interannual variation in deciduous broadleaf forest phenology using Landsat TM/ETM+ data. Remote Sensing of Environment, 132, 176-185

Olsson, P.-O., Lindström, J., \& Eklundh, L. (2016). Near real-time monitoring of insect induced defoliation in subalpine birch forests with MODIS derived NDVI. Remote Sensing of Environment, 181, 42-53 
This preprint has been submitted to Remote Sensing of Environment for peer review

822

823

824

825

826

827

828

829

830

831

832

833

834

835

836

837

838

839

840

841

842

843

844

845

846

847

848

849

850

851

852

853

854 855

856

857

858

859

860

861

Paritsis, J., \& Veblen, T.T. (2011). Dendroecological analysis of defoliator outbreaks on Nothofagus pumilio and their relation to climate variability in the Patagonian Andes. Global change biology, $17,239-253$

Pasquarella, V., Bradley, B., \& Woodcock, C. (2017). Near-Real-Time Monitoring of Insect Defoliation Using Landsat Time Series. Forests, 8, 275

Pasquarella, V., Elkinton, J.S., \& Bradley, B.A. (2018). Extensive gypsy moth defoliation in Southern New England characterized using Landsat satellite observations. Biol. Invasions, 20, 3047-3053

Pasricha, G.K. (2006). Kalman Filter and its Economic Applications

Pechony, O., \& Shindell, D.T. (2010). Driving forces of global wildfires over the past millennium and the forthcoming century. Proceedings of the National Academy of Sciences, 107, 19167-19170

Preisler, H.K., Hicke, J.A., Ager, A.A., \& Hayes, J.L. (2012). Climate and weather influences on spatial temporal patterns of mountain pine beetle populations in Washington and Oregon. Ecology, 93, 2421-2434

Rogan, J., \& Mietkiewicz, N. (2015). Land cover change detection. Land Resources Monitoring, Modeling, and Mapping with Remote Sensing; Thenkabail, PS, Ed, 579-603

Schmidt, S.F. (1981). The Kalman filter - Its recognition and development for aerospace applications. $J$. Guid. Control Dyn., 4, 4-7

Sedano, F., Kempeneers, P., \& Hurtt, G. (2014). A Kalman Filter-Based Method to Generate Continuous Time Series of Medium-Resolution NDVI Images. Remote Sensing, 6, 12381-12408

Seidl, R., Thom, D., Kautz, M., Martin-Benito, D., Peltoniemi, M., Vacchiano, G., Wild, J., Ascoli, D., Petr, M., \& Honkaniemi, J. (2017). Forest disturbances under climate change. Nature climate change, 7, 395-402

Townsend, P.A., Eshleman, K.N., \& others (2004). Remote sensing of gypsy moth defoliation to assess variations in stream nitrogen concentrations. Ecological

Turner, M.G., Baker, W.L., Peterson, C.J., \& Peet, R.K. (1998). Factors influencing succession: lessons from large, infrequent natural disturbances. Ecosystems, 1, 511-523

United States Department of, A. (2014). West Fork Fire Complex

Verbesselt, J., Hyndman, R., Newnham, G., \& Culvenor, D. (2010a). Detecting trend and seasonal changes in satellite image time series. Remote Sensing of Environment, 114, 106-115

Verbesselt, J., Hyndman, R., Zeileis, A., \& Culvenor, D. (2010b). Phenological change detection while accounting for abrupt and gradual trends in satellite image time series. Remote Sensing of Environment, 114, 2970-2980

Verbesselt, J., Zeileis, A., \& Herold, M. (2012). Near real-time disturbance detection using satellite image time series. Remote Sensing of Environment, 123, 98-108

Verdin, K.L., Dupree, J.A., \& Stevens, M.R. (2013). Postwildfire debris-flow hazard assessment of the area burned by the 2013 West Fork Fire Complex, southwestern Colorado. US Department of the Interior, US Geological Survey

Vicente-Guijalba, F., Martinez-Marin, T., \& Lopez-Sanchez, J.M. (2014). Crop Phenology Estimation Using a Multitemporal Model and a Kalman Filtering Strategy. IEEE Geoscience and Remote Sensing Letters, 11, 1081-1085 
This preprint has been submitted to Remote Sensing of Environment for peer review

862

863

864

865

866

867

868

869

870

871

872

873

874

875

876

877

878

879

880

881

882

883

884

885

886

887

888

889

890

891

892

893

894

895

Vogelmann, J.E., Gallant, A.L., Shi, H., \& Zhu, Z. (2016). Perspectives on monitoring gradual change across the continuity of Landsat sensors using time-series data. Remote Sensing of Environment, $185,258-270$

Walters, S.P., Schneider, N.J., \& Guthrie, J.D. (2011). Geospatial Multi-Agency Coordination (GeoMAC) wildland fire perimeters, 2008. Data Series

Westerling, A.L. (2016). Increasing western US forest wildfire activity: sensitivity to changes in the timing of spring. Phil. Trans. R. Soc. B, 371, 20150178

Woodcock, C.E., Allen, R., Anderson, M., Belward, A., Bindschadler, R., Cohen, W., Gao, F., Goward, S.N., Helder, D., \& Helmer, E. (2008). Free access to Landsat imagery. Science, 320, 1011-1011

Ye, S., Rogan, J., \& Sangermano, F. (2018). Monitoring rubber plantation expansion using Landsat data time series and a Shapelet-based approach. ISPRS J. Photogramm. Remote Sens., 136, 134-143

Zhao, K., Wulder, M.A., Hu, T., Bright, R., Wu, Q., Qin, H., Li, Y., Toman, E., Mallick, B., Zhang, X., \& Brown, M. (2019). Detecting change-point, trend, and seasonality in satellite time series data to track abrupt changes and nonlinear dynamics: A Bayesian ensemble algorithm. Remote Sens. Environ., 232, 111181

Zhu, Z. (2017). Change detection using landsat time series: A review of frequencies, preprocessing, algorithms, and applications. ISPRS Journal of Photogrammetry and Remote Sensing, 130, 370384

Zhu, Z. (2019). Science of Landsat Analysis Ready Data. Remote Sensing, 11, 2166

Zhu, Z., \& Woodcock, C.E. (2012). Object-based cloud and cloud shadow detection in Landsat imagery. Remote Sensing of Environment, 118, 83-94

Zhu, Z., \& Woodcock, C.E. (2014a). Automated cloud, cloud shadow, and snow detection in multitemporal Landsat data: An algorithm designed specifically for monitoring land cover change. Remote Sensing of Environment, 152, 217-234

Zhu, Z., \& Woodcock, C.E. (2014b). Continuous change detection and classification of land cover using all available Landsat data. Remote Sensing of Environment, 144, 152-171

Zhu, Z., Woodcock, C.E., Holden, C., \& Yang, Z. (2015). Generating synthetic Landsat images based on all available Landsat data: Predicting Landsat surface reflectance at any given time. Remote Sensing of Environment, 162, 67-83

Zhu, Z., Woodcock, C.E., \& Olofsson, P. (2012). Continuous monitoring of forest disturbance using all available Landsat imagery. Remote Sensing of Environment, 122, 75-91

Zhu, Z., Zhang, J., Yang, Z., Aljaddani, A.H., Cohen, W.B., Qiu, S., \& Zhou, C. (2019). Continuous monitoring of land disturbance based on Landsat time series. Remote Sensing of Environment 